\title{
Conformations of the Pyranoid Sugars. III. Infrared Absorption Spectra of Some Acetylated Aldopyranosides
}

\author{
R. Stuart Tipson and Horace S. Isbell
}

\author{
(May 5, 1960)
}

\begin{abstract}
The infrared absorption spectra of twenty-four acetylated aldopyranosides in the range of 5,000 to $250 \mathrm{~cm}^{-1}$ are reported. The conformation adopted by each of seventeen of the corresponding unacetylated glycosides had previously been assigned by us from a study of their infrared spectra. Analysis of the spectra revealed, for the acetylated glycosides (as for the parent glycosides), groups of absorption bands which showed a concerted shift on change of anomeric disposition. Assignment of conformation by the methods developed earlier led to the conclusion that each acetylated glycoside has the same conformation as its parent glycoside.

Intercomparison of the spectra of four of the remaining acetates with those of related acetates, especially in regard to the characteristic groups of absorption bands, afforded evidence that the anomeric methoxyl group is axial in methyl hepta- $O$-acetyl-4- $O-\beta$-Dglucopyranosyl- $\alpha$-D-mannopyranoside, and equatorial in methyl tetra- $O$-acetyl- $\beta$-D-mannopyranoside, methyl penta- $O$-acetyl-D-glycero- $\beta$-L-manno-heptopyranoside, and methyl hepta$O$-acetyl-4-O- $\beta$-D-glucopyranosyl- $\beta$-D-mannopyranoside. For lack of spectra of related acetylated aldopyranosides, assignments cannot yet be made for methyl hepta-O-acetyl6 - $O$ - $\beta$-D-glucopyranosyl- $\beta$-D-glucopyranoside, methyl penta- $O$-acetyl-D-glycero- $\beta$-D-ido-heptopyranoside, and methyl hepta- $O$-acetyl-4- $O-\beta$-D-galactopyranosyl- $\beta$-D-altropyranoside.
\end{abstract}

\section{Scope and Purpose of the Project}

The purpose of the present project was twofold. The first objective was to record the infrared absorption spectra of a variety of acetylated aldopyranosides for use in the identification of supposedly identical samples. The spectra of three of the compounds included in the present study were recorded by Kuhn $[1]^{1}$ for a limited range $\left(1,250\right.$ to $\left.667 \mathrm{~cm}^{-1}\right)$. In the following year, the spectra of 13 of the compounds were given (for the range of 5,000 to 667 $\mathrm{cm}^{-1}$ ) by Isbell and coworkers in a privately circulated report [2] which was subsequently published [3]. Twelve of the spectra obtained by these investigators were later discussed by Whistler and House [4], who noted certain bands in the range of 1,205 to $855 \mathrm{~cm}^{-1}$. Next, Barker and coworkers [5] examined the spectra of two of Kuhn's compounds and of four others that are included in the present study, but have not published them in sufficient detail to permit comparison over a wide spectral range; some of the bands in the range of 960 to $730 \mathrm{~cm}^{-1}$ were discussed. In the present article, the infrared absorption spectra of 24 acetylated aldopyranosides, in the range of 5,000 to $250 \mathrm{~cm}^{-1}$, are given; all of the bands of all of these compounds have been measured and have received consideration.

The second ebjective was the discovery of correlations that might be of value in structural analysis, both as regards (a) the presence of certain functional groups and (b) the particular conformation assumed by each compound. Isbell and coworkers [3] recorded the infrared spectra of 13 of the com-

Figures in brackets indicate the literature references at the end of this paper. The references for table 1 are given at the end of the table. pounds dissolved in suitable solvents, and were able to reach certain conclusions regarding correlations of the kind mentioned. However, at the low concentrations they employed, minor bands were absent or difficult to detect. The spectrograms have now been recorded for the solid phase, as pellets consisting of crystalline material suspended in an alkali-metal halide. [Such spectra show more bands than those for the same compounds dissolved in a solvent. This is because, for the solid, the following factors are operative: (1) A removal of degeneracies by perturbations from lattice forces; (2) occurrence of combination vibrations between molecular and lattice modes in the solid; and (3) an intensification of overtones and combination vibrations.]

In part II of this series of articles [6], a method was described for gaining information regarding the conformations of unsubstituted aldopyranosides from analysis of their infrared spectra. The analysis revealed groups of absorption bands, characteristic for each group-configuration, which displayed a concerted shift on change of anomeric disposition. This empirical observation was employed, in conjunction with a consideration of instability factors (arrived at on theoretical grounds), in making conformational assignments and in deciding the arrangement of groups (e.g., axial or equatorial) at the anomeric carbon atom of these and related compounds. The results were essentially in agreement with those obtained experimentally by Reeves [7] from a study of the behavior of these unsubstituted aldopyranosides on forming complexes with the cuprammonium reagent. The present article describes the results obtained on applying the same kind of analysis to the infrared spectra of the fully acetylated derivatives 
of 17 of the aldopyranosides studied in part II and of 7 acetylated aldopyranosides whose unacetylated parents were not included therein.

\section{Compounds Investigated}

Table 1 gives a list of the compounds, their code numbers [8], and an index to the spectrograms; the serial number of a compound is the same as the number of its spectrogram. Also included in table 1 are (a) the conformation (where known) of the corresponding unacetylated glycoside, as determined in the preceding paper in this series [6], and (b) assignments of conformations to the acetylated glycosides. The conformations are indicated by the system devised by Isbell and Tipson [9, 10].

The spectra were measured in the region of 5,000 to $667 \mathrm{~cm}^{-1}$ (sodium chloride optics) and in the region of 667 to $250 \mathrm{~cm}^{-1}$ (cesium bromide optics). The spectrograms are given together with a discussion of (a) the structure of the compounds and (b) some of the outstanding features of their spectra.

All of the compounds listed in table 1 are fully acetylated glycosides of aldoses, and all have the pyranoid ring. As a common structural feature, all but one of the glycosides have a glycosidic methoxyl group; one has a glycosidic cyclohexyloxy group. The acetylated glycosides differ in regard to one or more of the following structural features: (a) The $\alpha$ or $\beta$ anomeric configuration at carbon atom 1 ; (b) the configurations of the other carbon atoms of the pyranoid ring (including $\mathrm{C} 5$ in the hexosides and heptosides); (c) the nature of the substituent, if any, at C5 (including the configuration at C6 of the heptosides); and (d) the nature of the substituent at the $\mathrm{C}-4$ and $\mathrm{C}-6$ hydroxyl groups.

TABLE 1. Compounds measured, stable conformations, and index to spectrograms

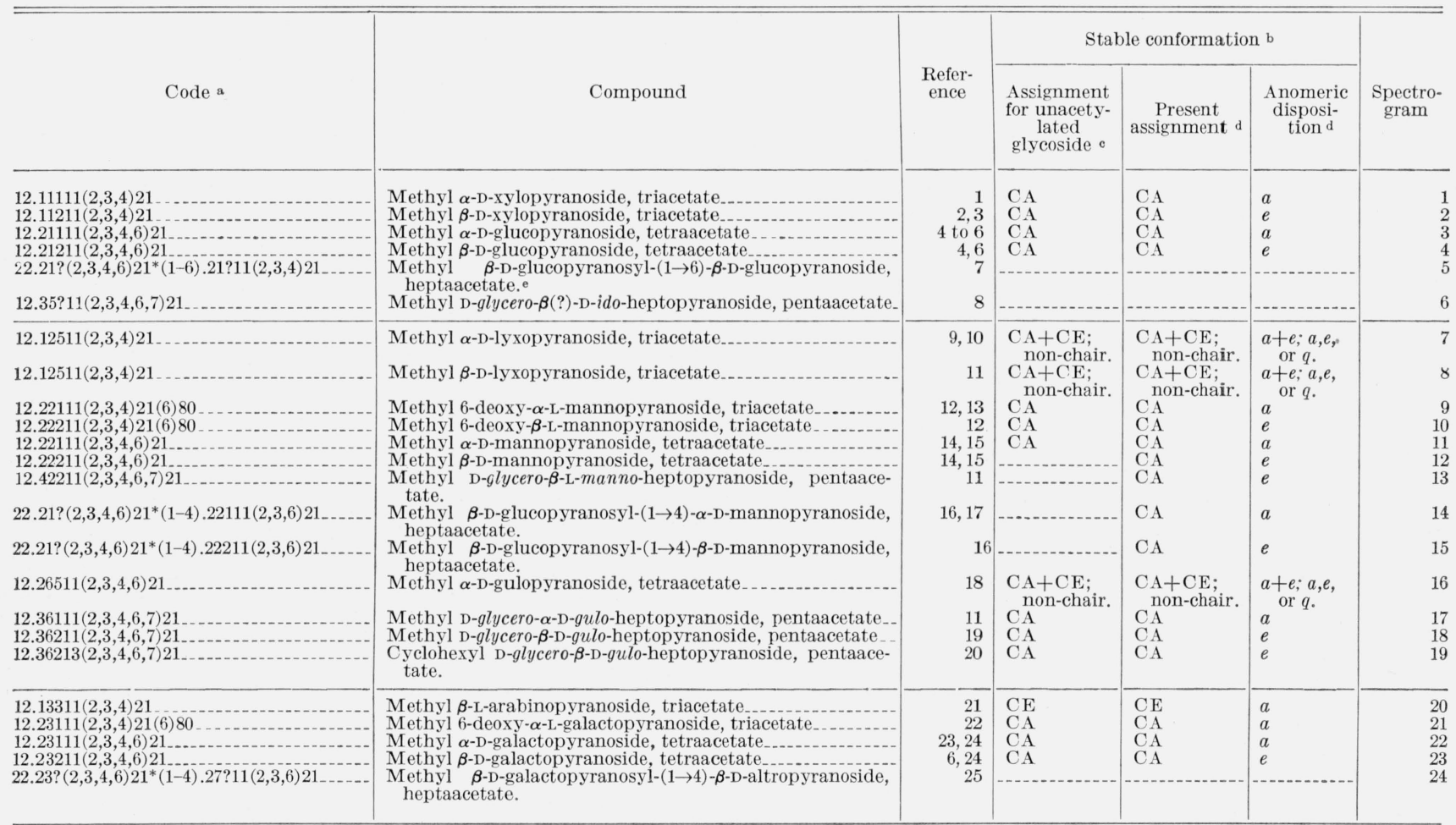

a The third figure after the point was inserted after the present conclusions as to conformation had been reached.

b Named by the system of H. S. Isbell and R. S. Tipson, Science 130, 793 (1959); J. Research NBS 64A, 171 (1960),

- Assignment made by R. S. Tipson and H. S. Isbell, J. Research NBS 64A, 239 (1960)

d After accepting several of the assignments for the unacetylated glycosides (see text).

e A sample of the original material, prepared by J. M. Johnson in November 1916, was kindly presented by N. K. Richtmyer.

\section{References for table 1}

1. C. S. Hudson and J. K. Dale, J. Am. Chem. Soc. 40, 997 (1918)

1. C. S. Hudson and J. K. Dale, J. Am. Chem. Soc. 40, 997 (1918)

2. C. S. Hudson and J. M. Johnson, J. Am. Chem.

4. C. S. Hudson and J. K. Dale, J. Am. Chem. Soc, 37, 1264 (1915).

5. E. Fischer and E. F. Armstrong, Ber. deut. chem. Ges. 34, 2890 (1901).

6. W. Koenigs and E. Knorr, Ber. deut. chem. Ges. 34, 957 (1901)

7. C. S. Hudson and J. M. Johnson, J. Am. Chem. Soc. 39, 1272 (1917).

8. H. L. Frush and H. S. Isbell, J. Research NBS 35, 111 (1945) RP1663.

9. P. A. Levene and M. L. Wolfrom, J. Biol. Chem. 78, 525 (1928); 79, 471 (1928)

10. F. P. Phelps and C. S. Hudson, J. Am. Chem. Soc. 50, 2049 (1928)

11. H. S. Isbell and H. L. Frush, J. Research NBS 24, 125 (1940) RP1274.

12. E. Fischer, M. Bergmann, and A. Rabe, Ber. deut. chem. Ges. 53, 2362 (1920).

13. M. Bergmann and H. Schotte, Ber. deut. chem. Ges. 54, 1569 (1921).
14. J. K. Dale, J. Am. Chem. Soc. 46, 1048 (1924)

14. J. K. Dale, J. Am. Chem. Soc. 46, 1048 (1924). Chem. Soc. 1932, 2108

16. T. L. Harris, E. L. Hirst, and C. E. Wood, J. Che

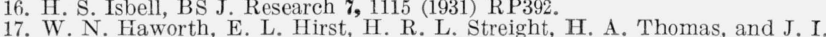
Webb, J. Chem. Soc. 1930, 2636

18. H. S. Isbell, BS J. Research 8, 1 (1932) RP396

19. W. N. Haworth, E. L. Hirst, and M. Stacey, J. Chem. Soc. 1931, 2864

20. E. Glaser and N. Zuckermann, Z. physiol. Chem. 166, 103 (1927)

21. C. S. Hudson and J. K. Dale, J. Am. Chem. Soc. 40, 992 (1918).

22. J. Minsaas, Rec. trav. chim. 56, 623 (1937).

23. F. Micheel and O. Littmann, Liebigs Ann. Chem. 466, 115 (1928).

24. J. K. Dale and C. S. Hudson, J. Am. Chem. Soc. 52, 2534 (1930)

25. H. L. Frush and H. S. Isbell, J. Research NBS 27, 113 (1941) RP1429. 


\section{Reference Aldopyranosides (of Known Conformation) and Their Acetates}

In part II of this series, the stable conformation of each of 21 aldopyranosides was deduced from an analysis of the respective infrared absorption spectrum, and was found to be in agreement with the assignment reached from a consideration of instability factors.

In the present study, the crystalline, fully acetylated derivatives of 17 of these aldopyranosides were available (group $\mathrm{A}$ ). In addition, 7 acetylated aldopyranosides (group B) whose unacetylated relatives had not been available (in the crystalline form) were examined. The spectra of the compounds comprising group A were analyzed, in order to determine if they were explicable on the basis that the acetate of a glycoside has the same conformation as its parent, unacetylated glycoside. The conclusions drawn from this study were then applied to deducing the stable conformation of members of group B.

\section{Classification of the Acetylated Glyco- sides Into Configurationally Related Groups}

The 24 compounds were classified into three groups; the members of each group have like configurational features.

\subsection{Acetylated Aldopyranosides of the xylo Configuration}

The members of this group of acetylated methyl aldopyranosides have the following general formula (I) for the CA conformation.

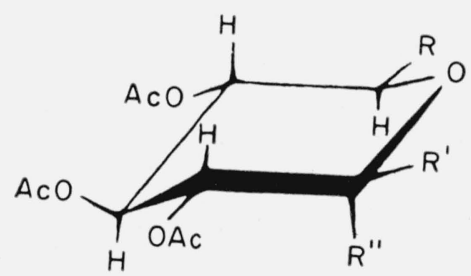

I

Compounds 1 to 5 , in this conformation, have the above general structure, with the following substituents.

1. Methyl $\alpha$-D-xylopyranoside triacetate, $\mathrm{R}=\mathrm{H}$; $\mathrm{R}^{\prime}=\dot{\mathrm{H}} ;$ and $\mathrm{R}^{\prime \prime}=\mathrm{OCH}_{3}$.

2. Methyl $\beta$-D-xylopyranoside triacetate, $\mathrm{R}=\mathrm{H}$; $\mathrm{R}^{\prime}=\mathrm{OCH}_{3} ;$ and $\mathrm{R}^{\prime \prime}=\mathrm{H}$.

3. Methyl $\alpha$-D-glucopyranoside tetraacetate, $\mathrm{R}$ $=\mathrm{CH}_{2} \mathrm{OAc} ; \mathrm{R}^{\prime}=\mathrm{H}$; and $\mathrm{R}^{\prime \prime}=\mathrm{OCH}_{3}$.

4. Methyl $\beta$-D-glucopyranoside tetraacetate, $\mathrm{R}$ $=\mathrm{CH}_{2} \mathrm{OAc} ; \mathrm{R}^{\prime}=\mathrm{OCH}_{3} ;$ and $\mathrm{R}^{\prime \prime}=\mathrm{H}$.

5. Methyl $\beta$-D-glucopyranosyl- $(1 \rightarrow 6)-\beta$-D-glucopyranoside heptaacetate, $\mathrm{R}=$ tetra- $O$-acetyl- $\beta$-D-glucopyranosyl- $\mathrm{OH}_{2} \mathrm{C} ; \mathrm{R}^{\prime}=\mathrm{OCH}_{3}$; and $\mathrm{R}^{\prime \prime}=\mathrm{H}$.
Compound 6 , if it is the $\beta$ anomer, has the following formulas (II) for the two chair-conformations.
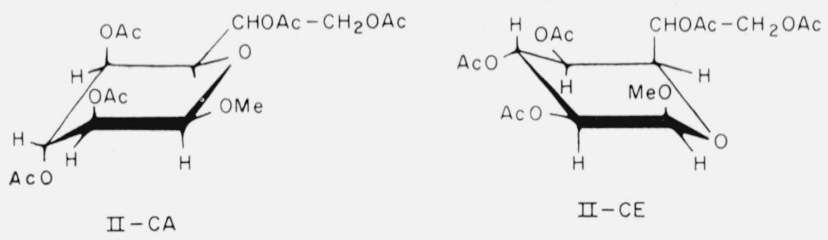

6. Methyl D-glycero- $\beta$-D-ido-heptopyranoside pentaacetate

\subsection{Acetylated Aldopyranosides of the 1yxo Configuration}

Six of the members of this group of configurationally related compounds have the D-lyxo or D-manno configuration and the following general formula (III) for the CA conformation.

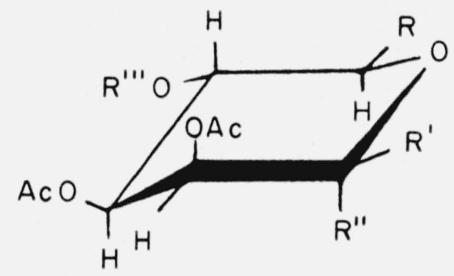

III

Compounds $7,8,11,12,14$, and 15 , in this conformation, have the above general structure, with the following substituents.

7. Methyl $\alpha$-D-lyxopyranoside triacetate, $\mathrm{R}=\mathrm{H}$; $\mathrm{R}^{\prime}=\mathrm{H} ; \mathrm{R}^{\prime \prime}=\mathrm{OCH}_{3} ;$ and $\mathrm{R}^{\prime \prime \prime}=\mathrm{Ac}$.

8. Methyl $\beta$-D-lyxopyranoside triacetate, $\mathrm{R}=\mathrm{H}$; $\mathrm{R}^{\prime}=\mathrm{OCH}_{3} ; \mathrm{R}^{\prime \prime}=\mathrm{H} ;$ and $\mathrm{R}^{\prime \prime \prime}=\mathrm{Ac}$.

11. Methyl $\alpha$-D-mannopyranoside tetraacetate, $\mathrm{R}$ $=\mathrm{CH}_{2} \mathrm{OAc} ; \mathrm{R}^{\prime}=\mathrm{H} ; \mathrm{R}^{\prime \prime}=\mathrm{OCH}_{3}$; and $\mathrm{R}^{\prime \prime \prime}=\mathrm{Ac}$.

12. Methyl $\beta$-D-mannopyranoside tetraacetate, $R$ $=\mathrm{CH}_{2} \mathrm{OAc} ; \mathrm{R}^{\prime}=\mathrm{OCH}_{3} ; \mathrm{R}^{\prime \prime}=\mathrm{H} ;$ and $\mathrm{R}^{\prime \prime \prime}=\mathrm{Ac}$.

14. Methyl $\beta$-D-glucopyranosyl- $(1 \rightarrow 4)-\alpha$-D-mannopyranoside heptaacetate, $\dot{\mathrm{R}}=\mathrm{CH}_{2} \mathrm{OAc} ; \mathrm{R}^{\prime}=\mathrm{H}$; $\mathrm{R}^{\prime \prime}=\mathrm{OCH}_{3} ;$ and $\mathrm{R}^{\prime \prime \prime}=$ tetra- $O$-acetyl- $\beta$-D-glucopyranosyl.

15. Methyl $\beta$-D-glucopyranosyl- $(1 \rightarrow 4)-\beta$-D-mannopyranoside heptaacetate, $\mathrm{R}=\mathrm{CH}_{2} \mathrm{OAc} ; \mathrm{R}^{\prime}$ $=\mathrm{OCH}_{3} ; \mathrm{R}^{\prime \prime}=\mathrm{H}$; and $\mathrm{R}^{\prime \prime \prime}=\operatorname{tetra}-O$-acetyl- $\beta$-Dglucopyranosyl.

Compounds 9, 10, and 13 have the L-manno configuration and the following general formula (IV) for the CA conformation.

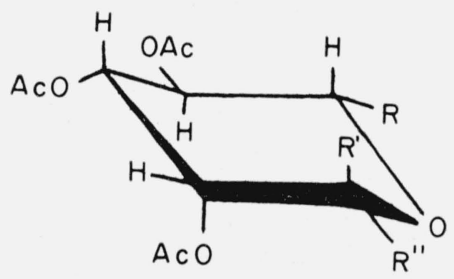

IV 
9. Methyl 6-deoxy- $\alpha$-L-mannopyranoside triacetate, $\mathrm{R}=\mathrm{CH}_{3} ; \mathrm{R}^{\prime}=\mathrm{OCH}_{3} ;$ and $\mathrm{R}^{\prime \prime}=\mathrm{H}$.

10. Methyl 6 -deoxy- $\beta$-L-mannopyranoside triacetate, $\mathrm{R}=\mathrm{CH}_{3} ; \mathrm{R}^{\prime}=\mathrm{H}$; and $\mathrm{R}^{\prime \prime}=\mathrm{OCH}_{3}$.

13. Methyl D-glycero- $\beta$-L-manno-heptopyranoside, $\mathrm{HCOAc}$ pentaacetate,

$\mathrm{R}$ is $\mathrm{CH}_{2} \mathrm{OAc} ; \mathrm{R}^{\prime}=\mathrm{H}$; and $\mathrm{R}^{\prime \prime}=\mathrm{OCH}_{3}$.

Compounds 16 to 19 have the D-gulo configuration and the following general formula $(V)$ for the CA o nfo rmation.

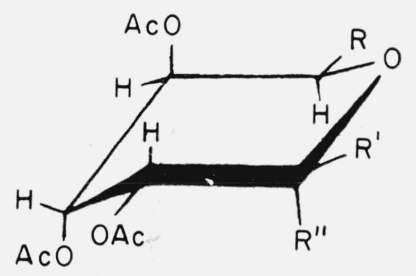

Z

16. Methyl $\alpha$-D-gulopyranoside tetraacetate, $\mathrm{R}=$ $\mathrm{CH}_{2} \mathrm{OAc} ; \mathrm{R}^{\prime}=\mathrm{H}$; and $\mathrm{R}^{\prime \prime}=\mathrm{OCH}_{3}$.

17. Methyl D-glycero- $\alpha$-D-gulo-heptopyranoside

\section{$\mathrm{HCOAc}$}

pentaacetate,

$\mathrm{R}$ is $\mathrm{CH}_{2} \mathrm{OAc} ; \mathrm{R}^{\prime}=\mathrm{H}$; and $\mathrm{R}^{\prime \prime}=\mathrm{OCH}_{3}$.

18. Methyl D-glycero- $\beta$-D-gulo-heptopyranoside

\section{$\mathrm{HCOAc}$}

pentaacetate,

$\mathrm{R}$ is $\mathrm{CH}_{2} \mathrm{OAc} ; \mathrm{R}^{\prime}=\mathrm{OCH}_{3}$; and $\mathrm{R}^{\prime \prime}=\mathrm{H}$.

19. Cyclohexyl D-glycero- $\beta$-D-gulo-heptopyranoside

$$
\text { HCOAc }
$$

pentaacetate,

$\mathrm{R}$ is $\mathrm{CH}_{2} \mathrm{OAc} ; \mathrm{R}^{\prime}=\mathrm{OC}_{6} \mathrm{H}_{11}$; and $\mathrm{R}^{\prime \prime}=\mathrm{H}$.

\subsection{Acetylated Aldopyranosides of the arabino Configuration}

The CE conformation of compound 20 and the CA conformation of compounds 22 and 23 are depicted in general formula VI.

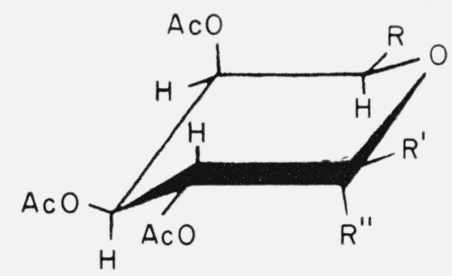

VI

20. Methyl $\beta$-L-arabinopyranoside triacetate, $\mathrm{R}=\mathrm{H}$; $\mathrm{R}^{\prime}=\mathrm{H}$; and $\mathrm{R}^{\prime \prime}=\mathrm{OCH}_{3}$.

22. Methyl $\alpha$-D-galactopyranoside tetraacetate, $\mathrm{R}=$ $\mathrm{CH}_{2} \mathrm{OAc} ; \mathrm{R}^{\prime}=\mathrm{H}$; and $\mathrm{R}^{\prime \prime}=\mathrm{OCH}_{3}$.
23. Methyl $\beta$-D-galactopyranoside tetraacetate, $\mathrm{R}=$ $\mathrm{CH}_{2} \mathrm{OAc} ; \mathrm{R}^{\prime}=\mathrm{OCH}_{3}$; and $\mathrm{R}^{\prime \prime}=\mathrm{H}$.

The CA conformation of compound 21 and the CE conformation of compound 24 are depicted in general formula VII.

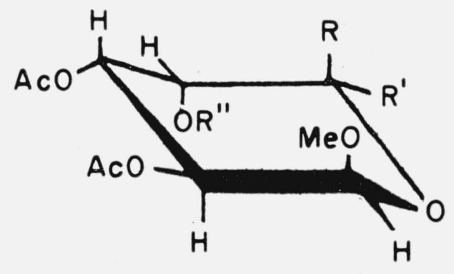

III

21. Methyl 6-deoxy- $\alpha$-L-galactopyranoside triacetate, $\mathrm{R}=\mathrm{H} ; \mathrm{R}^{\prime}=\mathrm{CH}_{3} ;$ and $\mathrm{R}^{\prime \prime}=\mathrm{Ac}$.

24. Methyl $\beta$-D-galactopyranosyl- $(1 \rightarrow 4)-\beta$-D-altropyranoside heptaacetate, $\mathrm{R}=\mathrm{CH}_{2} \mathrm{OAc} ; \mathrm{R}^{\prime}=\mathrm{H}$; and $\mathrm{R}^{\prime \prime}=$ tetra-O-acetyl- $\beta$-D-galactopyranosyl.

\section{Discussion of the Spectra}

In the present study, the positions of the various absorption bands for each of 24 acetylated aldopyranosides have been determined; the relative intensities of absorption were not examined in detail. The bands were compiled, and were studied by statistical and comparative methods, as previously described [11].

The conformations of 17 of the corresponding unacetylated glycosides had previously been assigned by us [6] from a study of their infrared absorption spectra (see table 1). Assignment was made on the basis of the empirical observation that, for the aldopentopyranosides and for members of any one groupconfiguration of the 5 - $C$-substituted aldopentopyranosides, a change of anomeric disposition was accompanied by a concerted shift of certain groups of absorption bands.

The same kind of examination has now been applied to the spectra of the fully acetylated derivatives of these 17 aldopyranosides. A similar effect was observed, namely, that certain groups of absorption bands show a concerted shift on change of anomeric disposition. Assignments were then made, on the basis of this empirical observation, for 15 of the acetylated derivatives, and were found to be in agreement with those previously made for the unacetylated relatives. Consequently, compounds 3 and 4 were assigned the $\mathrm{CA}$ conformation, which had previously been inferred for their parent glycosides [6].

The remaining seven spectra were those of acetylated glycosides whose parent glycosides had received no conformational assignment. Intercomparison of the spectra of four of these acetates (compounds 12 to 15 , table 1 ) with those of related acetates (especially in regard to the characteristic, anomerdifferentiating absorption bands) was then under- 
taken. For lack of spectra of related acetates, assignments could not be made for the remaining three compounds (see table 1 ; compounds 5, 6, and 24).

\subsection{Absorption Bands Possibly Indicative of the Disposition of Groups at the Anomeric Carbon Atom of the Methyl Tri-O-acetyl-aldopentopyrano- sides}

As the starting point in these analyses, we selected methyl tri- $O$-acetyl- $\beta$-D-xylopyranoside (compound 2 ) because, if this compound adopts a chair conformation, the reference groups will either be all axial (CE) or all equatorial (CA). Its spectrum was compared with that of its $\alpha$ anomer (compound 1), in order to determine the effect, on the spectrum, of changing the anomeric group from equatorial to axial, or vice versa. A comparison was then made with the spectrum of methyl tri- $O$-acetyl- $\beta$-L-arabinopyranoside (compound 20).

Bands that are essentially the same for these three compounds are given ${ }^{2}$ in table 2 . It seemed reasonable to assume tentatively that bands shown by all of these acetylated glycosides might be independent of total configuration, whereas those shown by one pair of anomers (e.g., compounds 1 and 2) having the same group-configuration might be a reflection, via shifting of bands, of an effect of the configuration of that pair.

${ }_{2}^{2}$ For comparison, bands (in about the same positions) shown by either or both anomers of methyl tri- $O$-acetyl-D-lyxopyranoside are also given in table 2.
In table 3 are given the bands shown by one anomer (but not by the other) of the methyl tri- $O$ acetyl-D-xylopyranosides and by methyl tri- $O$-acetyl$\beta$-L-arabinopyranoside. If the "anomer-differentiating" bands have any relationship to the axial or equatorial disposition of the respective glycosidic

TABLE 3. Bands shown by only one anomer of the methyl tri-O-acetyl-D-xylopyranosides (compounds 1 and 2) and by methyl tri-O-acetyl- $\beta$-L-arabinopyranoside (20), compared with bands for both anomers of methyl tri-O-acetyl-D-lyxopyranoside ( 7 and 8 )

\begin{tabular}{|c|c|c|c|c|}
\hline 1 & 20 & 2 & 7 & 8 \\
\hline 2933 & 2941 & & 2941 & \\
\hline $\begin{array}{r}2841 \\
\end{array}$ & 2849 & & 2849 & \\
\hline$\therefore 1205$ & $\begin{array}{l}\text { a } 1202 \\
051\end{array}$ & & ... & 1209 \\
\hline $\begin{array}{l}949 \\
921\end{array}$ & $\begin{array}{l}951 \\
926\end{array}$ & & 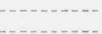 & \\
\hline 738 & 757 & & 771 & 755 \\
\hline 659 & 660 & & 668 & 665 \\
\hline 607 & & & 606 & 608 \\
\hline $\begin{array}{l}366 \\
358\end{array}$ & $\begin{array}{l}365(?) \\
359\end{array}$ & & & \\
\hline 350 & $\begin{array}{l}5030 \\
350\end{array}$ & & & $351(?)$ \\
\hline \multirow[t]{9}{*}{263} & $284(?)$ & 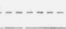 & & \\
\hline & & 3012 & & \\
\hline & & 2967 & & 2967 \\
\hline & & $\begin{array}{l}2890 \\
2874\end{array}$ & 2899 & 2865 \\
\hline & 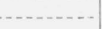 & 1754 & & 1745 \\
\hline & & 1403 & 1399 & \\
\hline & & & 1233 & \\
\hline & & 1155 & & 1148 \\
\hline & & & & \\
\hline
\end{tabular}

a See footnote $\mathrm{b}$ of table 2 .

TABLE 2. Bands $\left(\mathrm{cm}^{-1}\right)$ shown by both anomers of methyl tri-O-acetyl-D-xylopyranoside (compounds 1 and 2 ) and by methyl tri-O-acetyl- $\beta$-L-arabinopyranoside (20), and positionally corresponding bands of the methyl tri-O-acetyl-D-lyxopyranosides ( 7 and 8 )

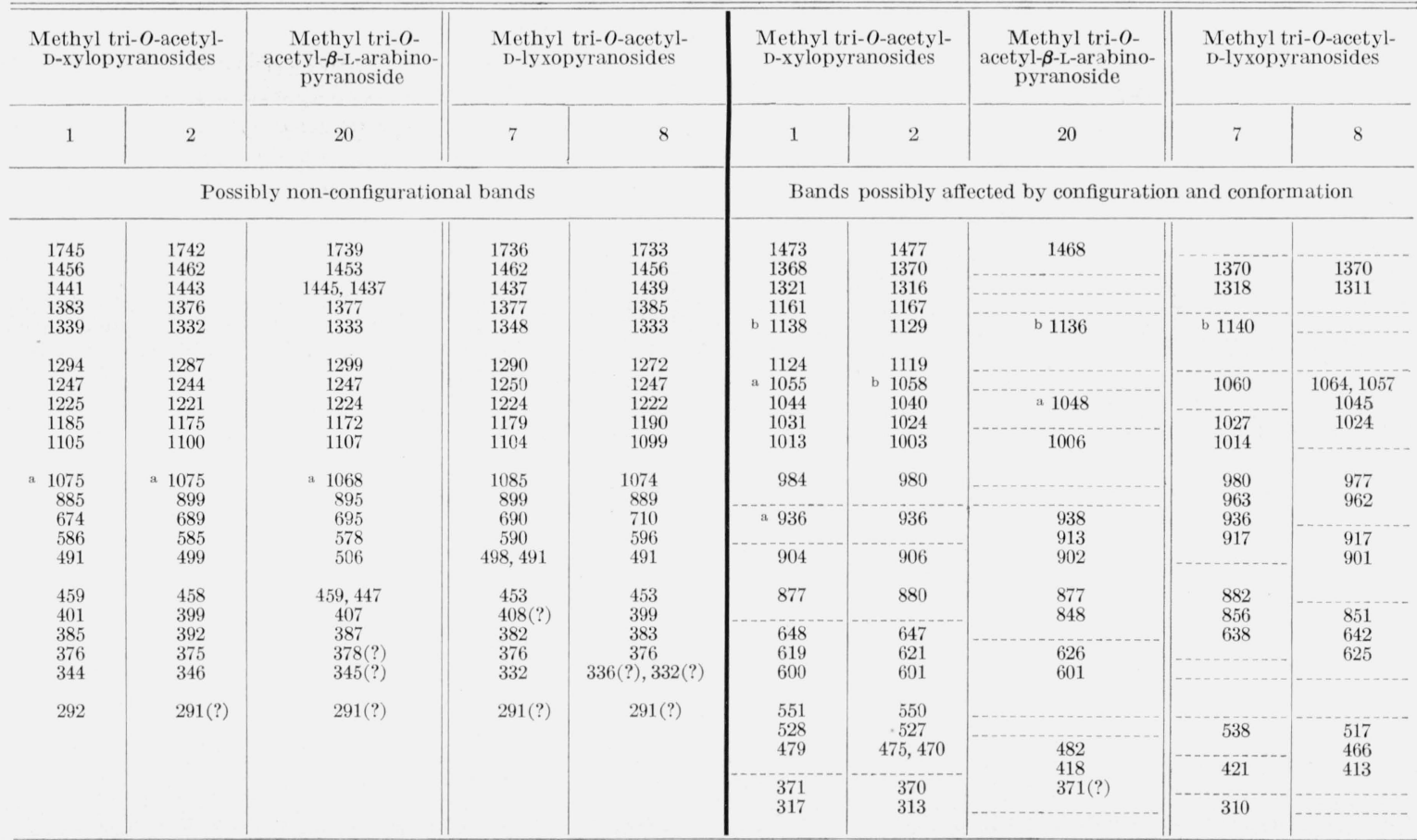

a These bands were mentioned by R. L. Whistler and L. R. House [4]

b These bands were mentioned by H. S. Isbell and coworkers [3]. 
methoxyl group, the results in table 3 clearly indicate that compounds 1 and 20 have the same anomeric disposition (that is, both have an equatorial, or both have an axial, methoxyl group), and that the anomeric disposition for compound 2 is different. If the assignment previously made [6] for the unacetylated relative of any one of these three acetylated glycosides is extended to its acetate, the conformations of the other two may be deduced from the results in table 3 . For example, if the anomeric group of methyl tri- $O$-acetyl- $\beta$-D-xylopyranoside is equatorial and that of its $\alpha$ anomer is axial, the results indicate that the anomeric group of methyl tri- $O$-acetyl- $\beta$ $\mathrm{L}$-arabinopyranoside is axial. These conclusions are in complete agreement with the assignments previously made [6] for the related unacetylated glycosides.

The corresponding bands of the methyl tri- $O$ acetyl-D-lyxopyranosides (compounds 7 and 8) are also given in table 3 . The results suggest that, for each anomer thereof, either (a) a conformation is adopted that is not a chair form and has a quasi, or a different kind of axial or equatorial, anomeric group, or (b) the crystalline material is a mixture of the $\mathrm{CA}$ and $\mathrm{CE}$ conformations. These conclusions agree with the previous findings for the unacetylated D-lyxopyranosides [6].

\subsection{Analysis of the Spectra of Groups of Con- figurationally Related, Acetylated Aldopyrano- sides, Excluding the Acetylated Aldopentopy- ranosides}

As a test of the method of analysis, an examination was made of the spectra of methyl tetra- $O$-acetyl$\alpha$-D-gulopyranoside (compound 16), the anomers of methyl penta-O-acetyl D-glycero-D-gulo-heptopyranoside (17 and 18), and cyclohexyl penta- $O$-acetylD-glycero- $\beta$-D-gulo-heptopyranoside (19), because assignments of anomeric disposition had previously

TABLE 4. Comparison a of absorption bands $\left(\mathrm{cm}^{-1}\right)$ shown by methyl tetra-O-acetyl- $\alpha$-D-gulopyranoside (compound 16), the anomers of methyl penta-O-acetyl-D-glycero-D-gulo-heptopyranoside (17 and 18), and cyclohexyl penta-O-acetyl-Dglycero- $\beta$-D-gulo-heptopyranoside (19)

\begin{tabular}{|c|c|c|c|c|c|c|c|}
\hline \multicolumn{2}{|c|}{ A } & \multicolumn{2}{|c|}{ B } & \multicolumn{2}{|c|}{ C } & \multicolumn{2}{|c|}{ D } \\
\hline $16(\alpha)$ & $17(\alpha)$ & $16(\alpha)$ & $18(\boldsymbol{\beta})$ & $17(\alpha)$ & $19(\beta)$ & $18(\beta)$ & $19(\boldsymbol{\beta})$ \\
\hline 2841 & 2849 & 1736 & 1745 & 831 & 825 & 2882 & 2865 \\
\hline $\begin{array}{l}1414 \\
1110\end{array}$ & $\begin{array}{l}1385 \\
1110\end{array}$ & $\begin{array}{l}943 \\
650\end{array}$ & $\begin{array}{l}943 \\
649\end{array}$ & & & $\begin{array}{l}1745 \\
1282\end{array}$ & $\begin{array}{l}1739 \\
1274\end{array}$ \\
\hline 818 & 831 & & & & & 1241 & 1242 \\
\hline & & & & & & $\begin{array}{l}943 \\
858 \\
649 \\
508 \\
472 \\
459 \\
445 \\
341\end{array}$ & $\begin{array}{l}942 \\
864 \\
645 \\
506 \\
473 \\
459 \\
443 \\
341\end{array}$ \\
\hline
\end{tabular}

a Key: A. Bands shown by compounds 16 and 17 , but not by 18 . B. Bands shown by compounds 16 and 18 , but not by 17 . C. Bands shown by compounds 17 and 19 , but not by 18 . D. Bands shown by compounds 18 and 19 , but not by 17 . been made [6] for the corresponding unacetylated glycosides. The results are given in table $4,{ }^{3}$ in which the anomer-differentiating bands shown by compounds 17 and 18 are compared with corresponding bands of compound 16 on the one hand and compound 19 on the other. Such bands, shown by two of the compounds (but not by the other two) are listed as follows: Column A, compounds 16 and 17, B, 16 and $18 ; \mathrm{C}, 17$ and $19 ; \mathrm{D}, 18$ and 19 . It may be seen that compound 16 shows about equal similarity to either compound 17 or 18 . In contrast, compound 17 has only one such band in common with compound 19 , but compounds 18 and 19 have twelve such bands in common. If the assignment previously made for the unacetylated parent is extended to the acetate, for any one of the acetates 17 , 18 , or 19 , the results are in accordance with the assignments previously made for all four parent glycosides. These are, for the anomeric group, as follows: Compound 17, axial; compounds 18 and 19, equatorial; and compound 16 , quasi, or a different kind of axial or equatorial disposition, or a mixture of axial and equatorial forms.

As a further check on the results accruing from our method of analysis, a second group of acetylated glycosides (for whose parent glycosides assignments had previously been made [6]) was studied. The results are given in table 5: column $\mathrm{A}$ lists three bands shown by methyl tetra- $O$-acetyl- $\beta$-D-galactopyranoside (compound 23) but not by compounds 21 and 22 ; column $\mathrm{B}$ lists eleven bands shown by methyl tri- $O$-a cet y l-6-d eo x y- $\alpha$-L-gal a c t op y ranoside (compound 21) and by the $\alpha$ anomer, compound 22 . Compounds 21 and 23 showed no anomer-differentiating bands in common. Consequently, if the assignment previously made for the unacetylated parent of any one of these three compounds is extended to the corresponding acetate, the assignments for the acetates are in harmony with those for the three parent glycosides. For the disposition of the ano-

3 To conserve space, bands which do not differentiate between anomers have been omitted from tables 4 to 9 .

TABLE 5. Comparison a of absorption bands $\left(\mathrm{cm}^{-1}\right)$ shown by the anomers of methyl tetra-O-acetyl-D-galactopyranoside (compounds 22 and 23) and by methyl tri-O-acetyl-6-deoxy$\alpha$-L-galactopyranoside (21)

\begin{tabular}{c|r|r}
\hline \hline \multirow{2}{*}{ A } & \multicolumn{2}{|c}{$\mathrm{B}$} \\
\cline { 3 - 3 } $23(\boldsymbol{\beta})$ & $21(\boldsymbol{\alpha})$ & $22(\boldsymbol{\alpha})$ \\
\cline { 3 - 3 } & & \\
\cline { 3 - 3 } 1014 & 2933 & 2941 \\
$\mathrm{~b} 880$ & 1215 & 1217 \\
611 & 1200 & c 1190 \\
& 1133 & c 1134 \\
& 1049 & 1041 \\
& 941 & b 949 \\
& 928 & b 936 \\
& 817 & b 821 \\
& 759 & b 758 \\
& 696 & 712 \\
& 487 & 490 \\
& &
\end{tabular}

a Key: A. Bands shown by compound 23, but not by compounds 21 and 22 . B. Bands shown by compounds 21 and 22 , but not by 23 .

b These bands were mentioned by S. A. Barker and coworkers [5]

c See footnotes $a$ and $b$ to table 2 . 
meric group, these are as follows: Compounds 21 and 22 , axial; and compound 23 , equatorial.

All of the foregoing deductions are compatible with the concept that an acetylated glycoside adopts the same conformation as its parent (unacetylated) glycoside. Consequently, because of the agreement between the assignments previously made for the parent glycosides [6] and now made for the acetylated glycosides, the utility of our empirical method for analyzing the spectra (and the validity of the deductions) was apparently established. The spectra of the remaining 12 glycosides were, therefore, examined. No assignment had previously been made as to the stable conformation of compounds $5,6,12$, $13,14,15$, and 24 .

No assignment could be made for compound 5 (in comparison with compounds 3 and 4) because of the lack of the $\alpha$ anomer (with respect to the glycosidic methyl group) of compound 5. However, the anomer-differentiating bands for the anomers of methyl tetra- $O$-acetyl-D-glucopyranoside (compounds 3 and 4) are presented in table 6 for future use.

No assignment could be made for compound 6 , because no other acetylated methyl aldopyranosides having the $i d o$ configuration were available for comparison. Similarly, no assignment could be made for compound 24, because of a lack of acetylated methyl aldopyranosides having the altro configuration.

Finally, assignments were sought for compounds 12 to 15 ; their spectra were compared with those of compounds 9 to 11 (for which we had previously made assignments for the unacetylated glycosides). First of all, the spectra of the anomers of methyl tri- $O$-acetyl-6-deoxy-L-mannopyranoside (compounds 9 and 10) were compared with those of the anomers of methyl tetra-O-acetyl-D-mannopyranoside (compounds 11 and 12). The results are given in table 7 ; column $\mathrm{A}$ gives bands shown by compounds 9 and 11 ( $\alpha$ anomers) but not by compounds 10 and 12 ( $\beta$ anomers), and column B records bands shown by the two $\beta$ anomers but not by the $\alpha$ anomers. (Only one discrepancy was noted, namely, that compounds 10 and 11 show a band at $1,168-1,167 \mathrm{~cm}^{-1}$ that is absent from the spectra of compounds 9 and 12.) It may be concluded that, assuming the validity of

TABLE 6. Bands $\left(\mathrm{cm}^{-1}\right)$ differentiating between the anomers of methyl tetra-O-acetyl-D-glucopyranoside (compounds 3 and 4)

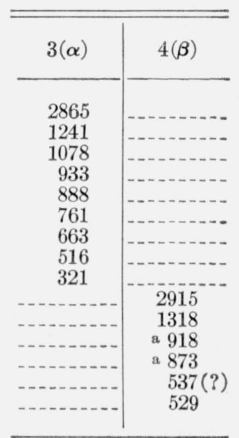

a See footnote b to table 5 .
TABLE 7. Comparison a of absorption bands $\left(\mathrm{cm}^{-1}\right)$ shown by the methyl tri-O-acetyl-6-deoxy-L-mannopyranosides (compounds 9 and 10) and by the methyl tetra-O-acetyl-D-mannopyranosides (11 and 12)

\begin{tabular}{|c|c|c|c|}
\hline \multicolumn{2}{|c|}{ A } & \multicolumn{2}{|c|}{ B } \\
\hline $9(\alpha)$ & $11(\alpha)$ & $10(\beta)$ & $12(\beta)$ \\
\hline $\begin{array}{r}1443 \\
\text { b } 884 \\
\text { b } 837 \\
\text { b } 797 \text {, b } 791 \\
685 \\
614\end{array}$ & $\begin{array}{r}1443 \\
\text { b } 886 \\
\text { b } 832 \\
\text { b } 792 \\
690 \\
616\end{array}$ & $\begin{array}{r}1410 \\
1076 \\
867 \\
752 \\
724 \\
412,406\end{array}$ & $\begin{array}{r}1404 \\
1074 \\
\text { b } 867 \\
\text { b } 752 \\
723 \\
408\end{array}$ \\
\hline & 510 & & \\
\hline
\end{tabular}

a Key: A. Bands shown by compounds 9 and 11, but not by compounds 10 and 12. B. Bands shown by compounds 10 and 12 , but not by compounds 9 and 11 . b see footnote b to table 5 .

the correlations, if the anomeric group of the $\alpha$ anomers is axial, that of the $\beta$ anomers is equatorial, or vice versa. If the conformation assigned to the unacetylated parent of any one of three of these four compounds $(9,10$, and 11$)$ is accepted for its acetate, the deduced conformations for the other two are in agreement with those for the unacetylated compounds. Furthermore, the results indicate that methyl tetra- $O$-acetyl- $\beta$-D-mannopyranoside (compound 12) has an equatorial anomeric group.

The anomeric disposition of methyl penta- $O$ acetyl-D-glycero- $\beta$-L-manno-heptopyranoside (13) was now studied by a double comparison-against (a) the anomers of methyl tri- $O$-acetyl-6-deoxy-Lmannopyranoside, and (b) the anomers of methyl tetra- $O$-acetyl-D-mannopyranoside. The results are given in table 8 . Column $\mathrm{A}$ of table 8 records bands shown by compounds 9 and 13 but not by 10 ; column B gives bands shown by compound 9 but not by 10 and 13 ; column $\mathrm{C}$ gives bands shown by 10 and 13 but not by 9 ; column $\mathrm{D}$ gives bands shown by 11 and 13 but not by 12 ; column E gives bands shown by compound 11 but not by 12 and 13 ; and column $\overline{\mathrm{F}}$ gives bands shown by 12 and 13 but not by 11 . It is seen that, whereas the 16 bands in columns B and C indicate similarity between compounds 10 and 13 , only two bands in column A suggest similarity of compounds 9 and 13 . Similarly, whereas the 18 bands in columns $\mathrm{E}$ and $\mathrm{F}$ indicate similarity between compounds 12 and 13 , only four bands in column D suggest similarity between compounds 11 and 13 . Thus, a total of 34 bands indicate relationship of compound 13 to compounds 10 and 12 (as against a total of six bands indicating a relationship of compound 13 to compounds 9 and 11). These results suggest that the anomeric group of compound 13 is equatorial.

To obtain information regarding the disposition of the methoxyl group in the anomers of methyl hepta$O$-a cetyl-4-O- $\beta$-D-glucopyranosyl-D-mannopyranoside (compounds 14 and 15), the spectra of these compounds were compared with those of the anomers of methyl tetra-O-acetyl-D-mannopyranoside (compounds 11 and 12). The results are given in table 9; column A records bands shown by compounds 11 
TABLE 8. Comparison a of absorption bands $\left(\mathrm{cm}^{-1}\right)$ shown by the methyl tri-O-acetyl-6-deoxy-L-mannopyranosides (compounds 9 and 10), the methyl tetra-O-acetyl-D-mannopyranosides (11 and 12), and methyl penta-O-acetyl-D-glycero- $\beta$-L-mannoheptopyranoside (13)

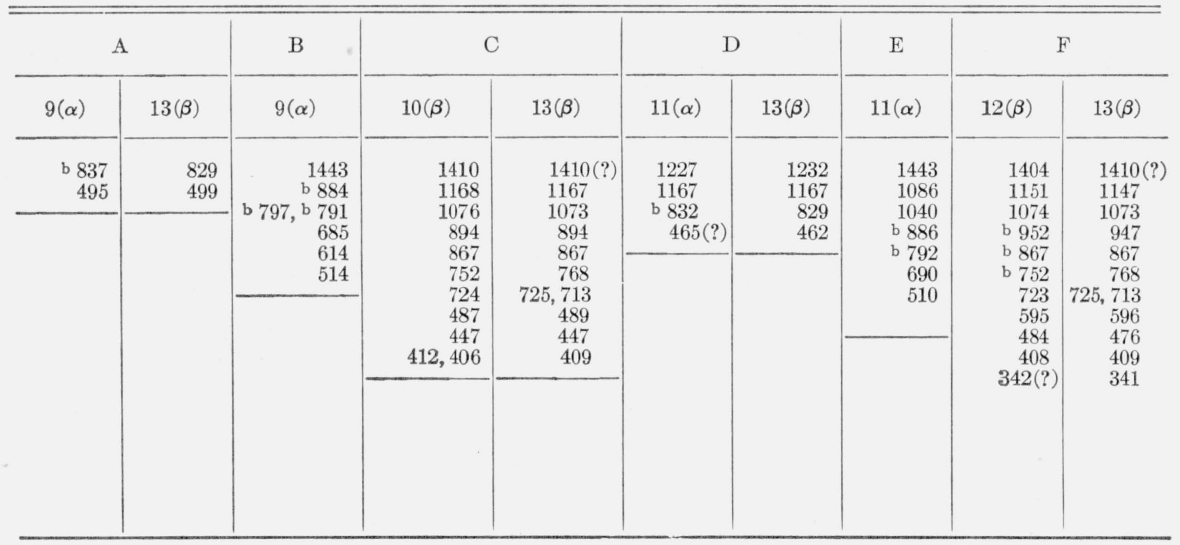

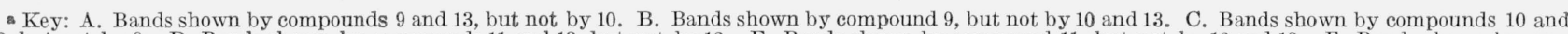

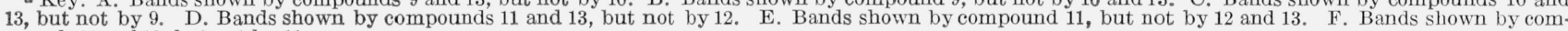
pounds 12 and 13 , but not by 11 .

b See footnote $b$ to table 5 .

TABLE 9. Comparisona of absorption bands $\left(\mathrm{cm}^{-1}\right)$ shown by the methyl tetra-O-acetyl-D-mannopyranosides (compounds 11 and 12) and by the methyl hepta-O-acetyl-4-O- $\beta$-Dglucopyranosyl-D-mannopyranosides (14 and 15)

\begin{tabular}{r|r|r|r}
\hline \hline \multicolumn{2}{c|}{ A } & \multicolumn{2}{|c}{ B } \\
\cline { 1 - 1 } $11(\boldsymbol{\alpha})$ & $14(\boldsymbol{\alpha})$ & \multicolumn{1}{|c|}{$12(\boldsymbol{\beta})$} & $15(\boldsymbol{\beta})$ \\
\cline { 1 - 1 } & & & \\
\cline { 1 - 2 } 1086 & 1083 & 1010 & 1003 \\
978 & 976 & b 952 & 952 \\
b 792 & 803 & b 904 & 904 \\
690 & 693 & b 752 & 750 \\
616 & 614 & 723 & 731 \\
559 & 557 & 570 & 567 \\
& & &
\end{tabular}

a Key: A. Bands shown by compounds 11 and 14 , but not by 12 and 15 . B Bands shown by compounds 12 and 15 , but not by 11 and 14 .

b See footnote b to table 5 .

and 14 (but not by compounds 12 and 15 ); column B gives the bands shown by compounds 12 and 15 (but not by compounds 11 and 14). If the assignments previously made for compounds 11 and 12 are accepted, these results indicate that the methoxyl group is axial in compound 14 and equatorial in compound 15 .

For molecules as complex as those of the acetylated aldopyranosides, many of the observed bands cannot yet be assigned to particular vibrational modes. Thus, in sections 5.1 and 5.2 , we have not been concerned with (a) which bands, arising from vibrations localized in a functional group, are relatively independent of the remainder of the molecule, or (b) which bands involve other parts of the molecule. However, in section 5.3, bands possibly attributable to specific functional groups are considered.

\subsection{Other Absorption Bands}

All of the compounds in this study are acetates, and their spectra all show at least one band $(\mathrm{C}=\mathrm{O}$ stretching frequency) at 1,764 to $1,736 \mathrm{~cm}^{-1}$ (except for compound 5, with a band at 1,773 $\left.\mathrm{cm}^{-1}\right)$; at 1,264 to $1,241 \mathrm{~cm}^{-1}$ (or at 1,259 to $1,239 \mathrm{~cm}^{-1}$ ); at 1,235 to $1,215 \mathrm{~cm}^{-1}$; at 667 to $632 \mathrm{~cm}^{-1}$; and at 614 to $585 \mathrm{~cm}^{-1}$. All of the spectra show at least one band at 2,994 to $2,941 \mathrm{~cm}^{-1}(\mathrm{C}-\mathrm{H}$ stretching $)$; and at 1,462 to $1,445 \mathrm{~cm}^{-1}$ and 1,335 to $1,318 \mathrm{~cm}^{-1}$ (C-H bending). All of the spectra show an absorption band at 1,445 to $1,431 \mathrm{~cm}^{-1}$ and at 1,379 to $1,368 \mathrm{~cm}^{-1}$, presumably caused by deformation of the $\mathrm{CH}_{3}$ groups.

Figure 1 gives the percentage of the 24 compounds in this study that show absorption bands in the various regions of the infrared spectrum; the crosshatched areas in the region of 333 to $250 \mathrm{~cm}^{-1}$ correspond to the region marked, on certain spectra, with dashes. For the range of 5,000 to $2,000 \mathrm{~cm}^{-1}$, decrements of $20 \mathrm{~cm}^{-1}$ in wavenumber were used; and, for the range of 2,000 to $250 \mathrm{~cm}^{-1}$, decrements of $10 \mathrm{~cm}^{-1}$. Figure 2 depicts the corresponding "profile" for 21 unacetylated glycosides, compiled from the spectra given in part II of this series [6].

Compound 19, having a cyclohexyloxy group, showed bands at 2,941 and $2,865 \mathrm{~cm}^{-1}$, possibly characteristic of $-\mathrm{CH}_{2}-(\mathrm{C}-\mathrm{H}$ stretching $)$. It also showed bands at 1,451 and $1,435 \mathrm{~cm}^{-1}$, possibly attributable to $-\mathrm{CH}_{2}-(\mathrm{C}-\mathrm{H}$ deformation $)$.

All of the acetylated methyl aldopyranosides show a band in the range of 2,882 to $2,833 \mathrm{~cm}^{-1}$, excepting compound 4 (band at $2,915 \mathrm{~cm}^{-1}$ ). This band may possibly be attributable to the glycosidic methoxyl group, since Henbest and coworkers [12] have observed that methoxyl groups absorb in the range of 2,832 to $2,819 \mathrm{~cm}^{-1}$. All of the acetylated methyl glycopyranosides also show bands at 1,374 to $1,325 \mathrm{~cm}^{-1}, 1,259$ to $1,241 \mathrm{~cm}^{-1}, 1,148$ to $1,114 \mathrm{~cm}^{-1}$, and 1,110 to $1,074 \mathrm{~cm}^{-1}$. A band near $1,100 \mathrm{~cm}^{-1}$ is characteristic [13] of the methoxyl groups in methoxysteroids. 


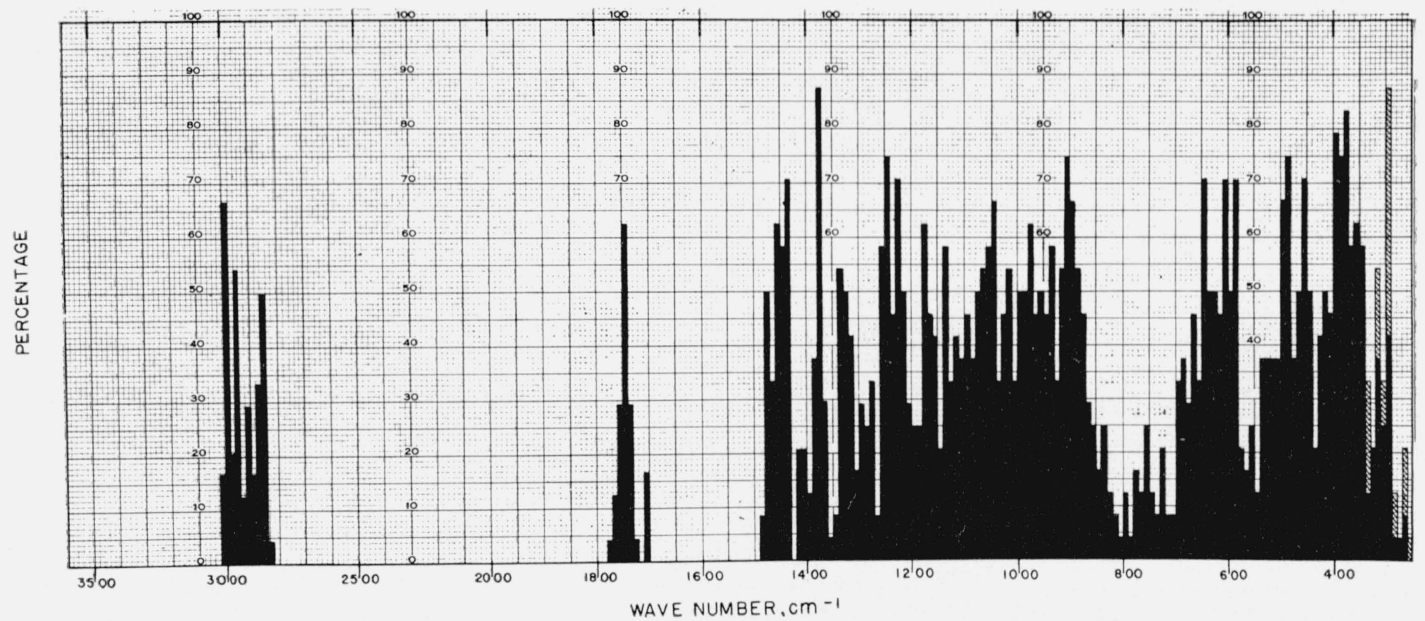

FIGURE 1. Percentage (of the 24 acetylated glycosides) which showed infrared absorption at the various regions of the infrared spectrum $\left(5,000\right.$ to $\left.250 \mathrm{~cm}^{-1}\right)$.

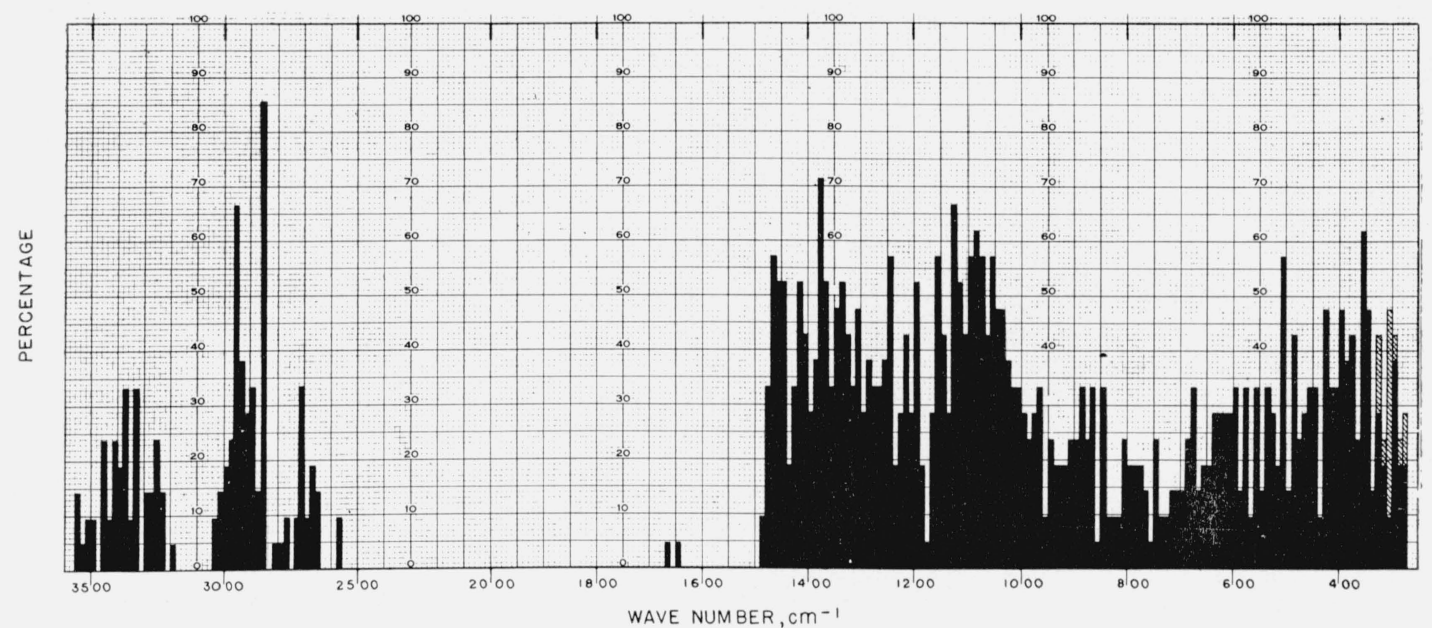

FIGURE 2. Percentage (of the 21 unacetylated glycosides) which showed infrared absorption at the various regions of the infrared spectrum $\left(5,000\right.$ to $\left.250 \mathrm{~cm}^{-1}\right)$.

\section{Experimental Procedures}

\subsection{Preparation and Purification of the Compounds}

The compounds listed in table 1 were prepared by the methods given in the references cited. Most of the compounds were prepared in the course of an earlier study [14] on the structural and configurational relationships of the anomers of the methyl pentopyranosides, hexopyranosides, and heptopyranosides. Each acetate was recrystallized from an appropriate solvent until further recrystallization caused no change in its melting point or optical rotation.

\subsection{Preparation of the Pellets}

Samples for spectrophotometric study were prepared in the solid phase, as pellets consisting of the crystalline acetate suspended in an alkali-metal halide, exactly as previously described [11]. For the range of 5,000 to $667 \mathrm{~cm}^{-1}$, a concentration of 0.4 $\mathrm{mg}$ of acetate per $100 \mathrm{mg}$ of potassium chloride was used, except for compounds $5(4 \mathrm{mg} / 100 \mathrm{mg})$ and 15 $(0.8 \mathrm{mg} / 100 \mathrm{mg})$. For the range of 667 to $250 \mathrm{~cm}^{-1}$, a concentration of $2 \mathrm{mg}$ of acetate per $100 \mathrm{mg}$ of potassium iodide was used, except for compounds 5 $(6 \mathrm{mg} / 100 \mathrm{mg})$ and $15(3 \mathrm{mg} / 100 \mathrm{mg})$. Comparisons of intensity of absorption, from one compound to another, can only be true and quantitative where the molar concentration is the same. 

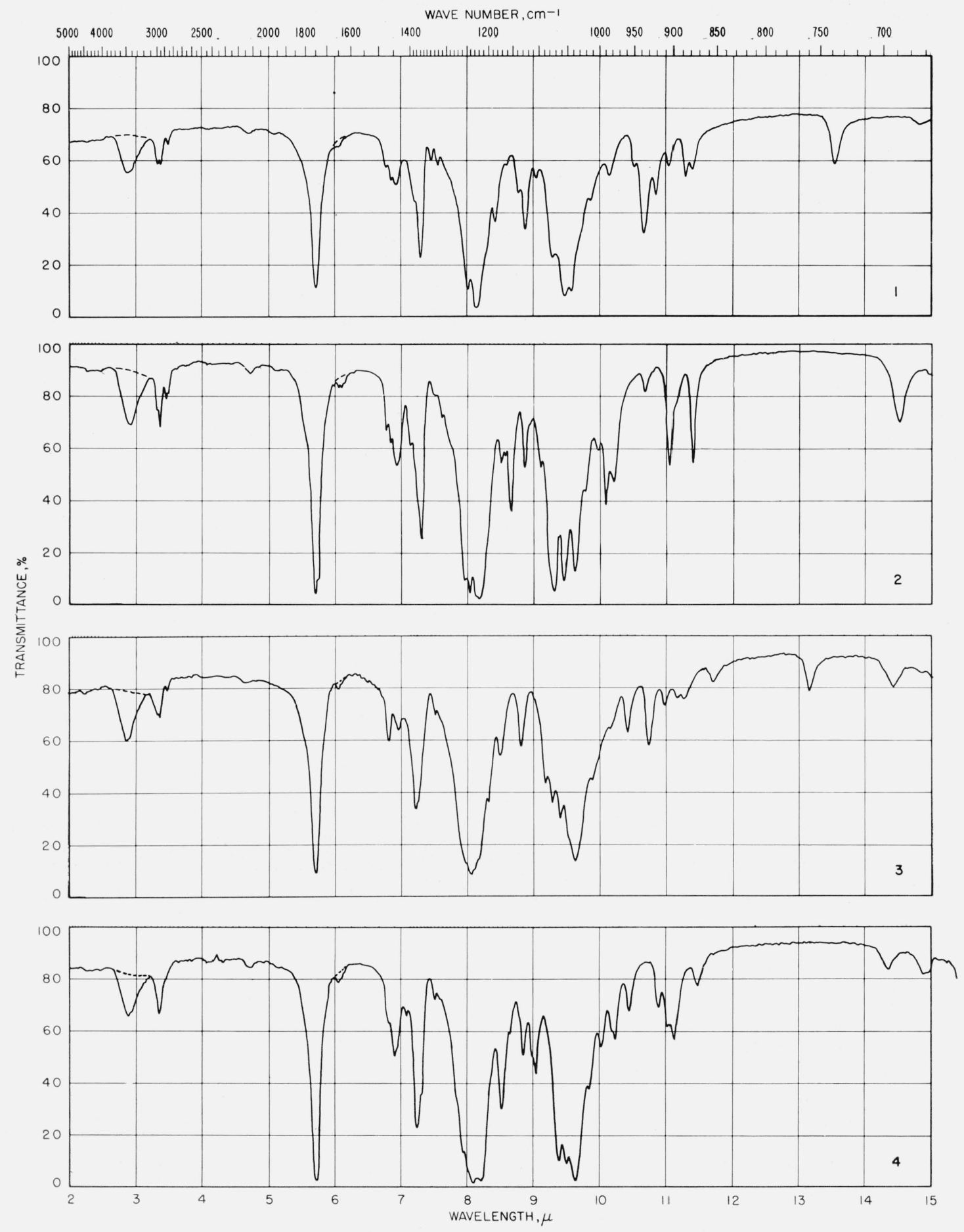

FIGURE 3. Spectrograms of materials in potassium chloride pellets.

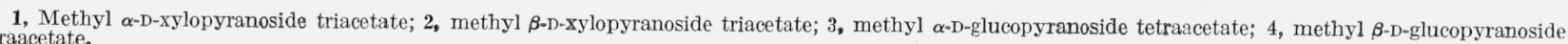
tetraacetate. 

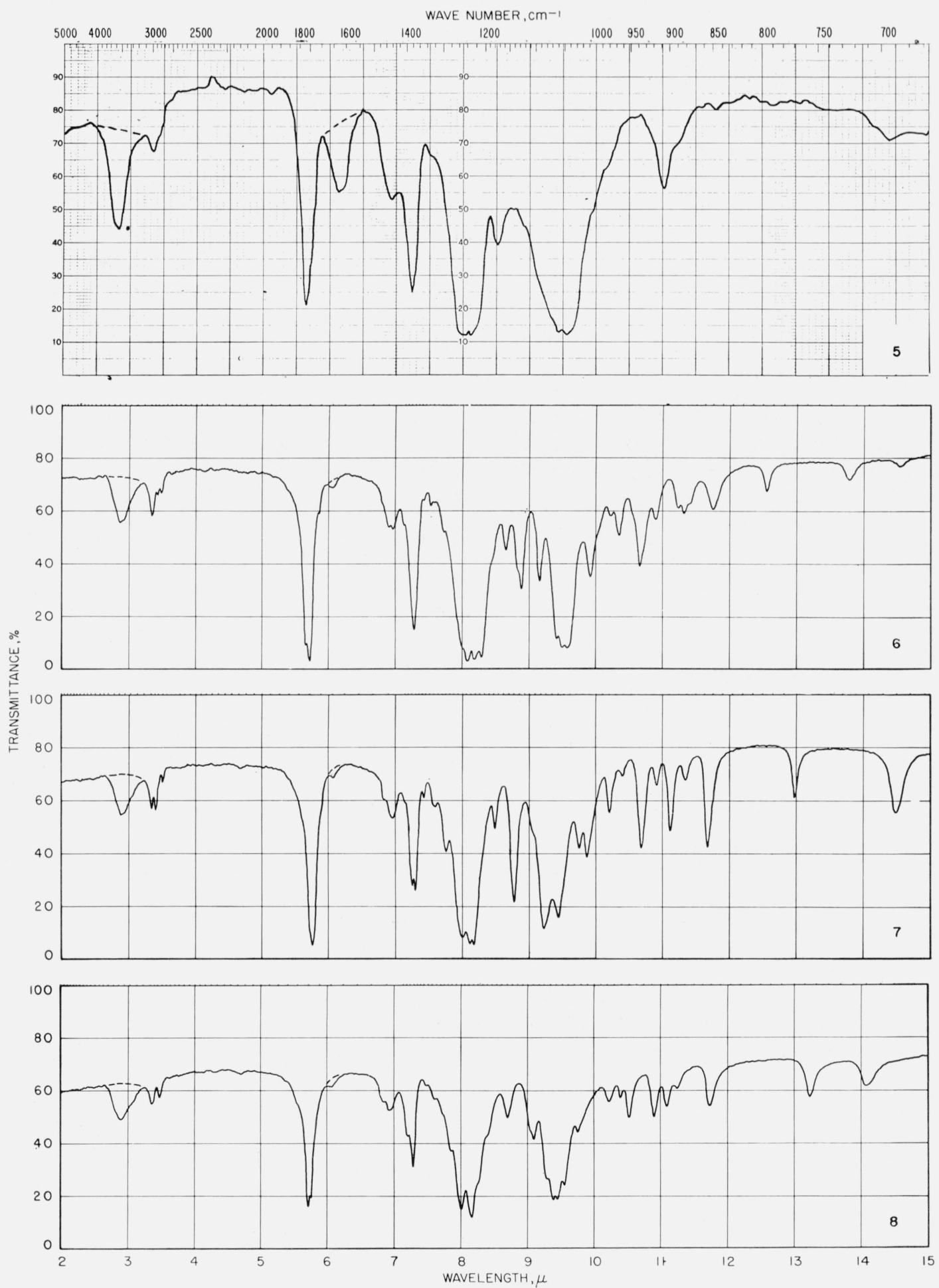

Figure 3. Spectrograms of materials in potassium chloride pellets.-Continued

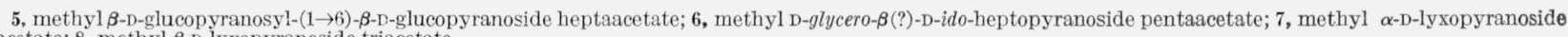
triacetate; 8 , methyl $\beta$-D-lyxopyranoside triacetate. 

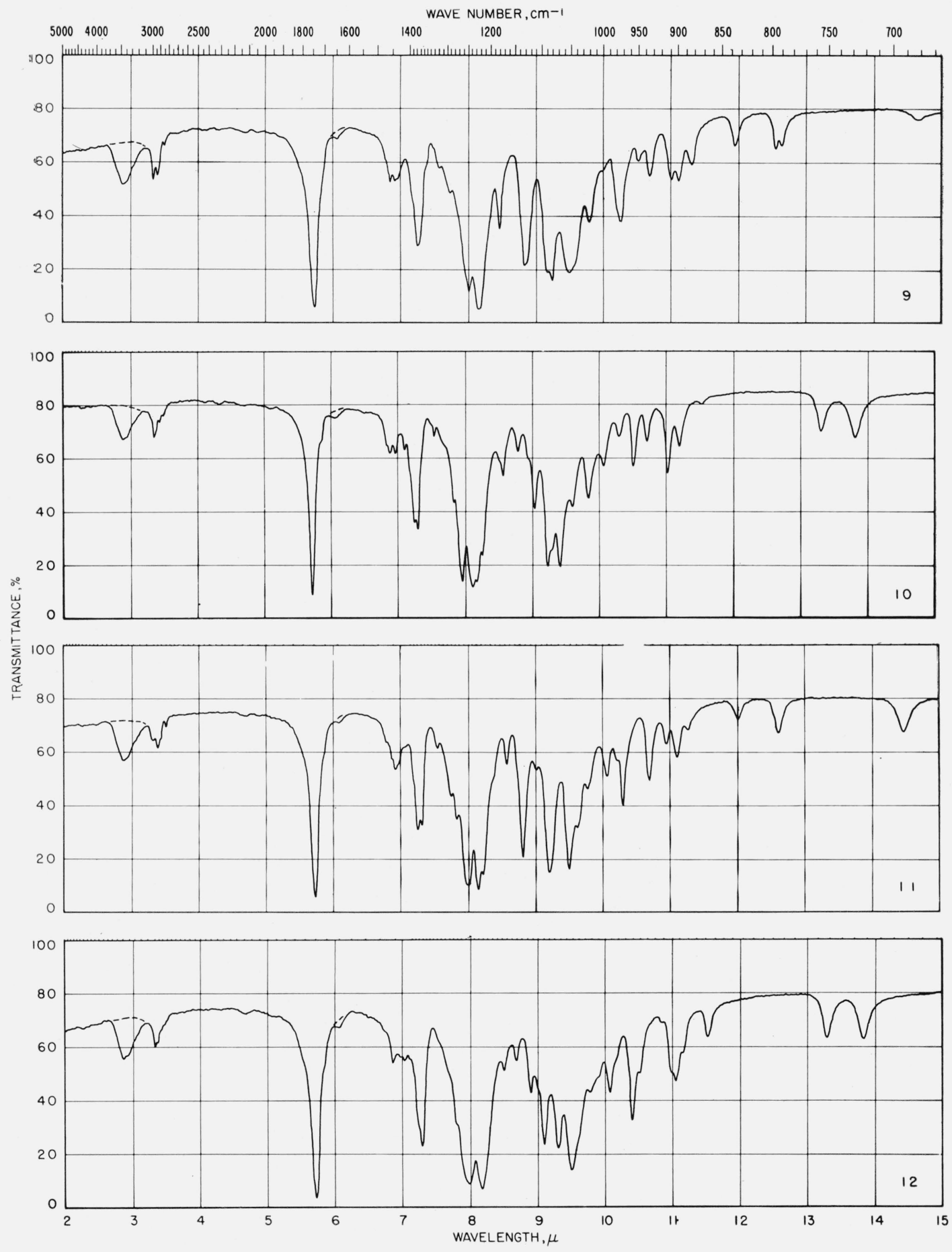

FIGURE 3. Spectrograms of materials in potassium chloride pellets.-Continued

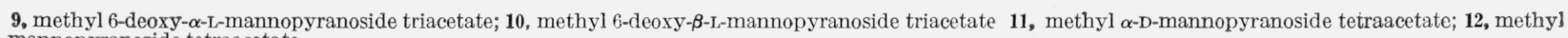
$\beta$-D-mannopyranoside tetraacetate. 

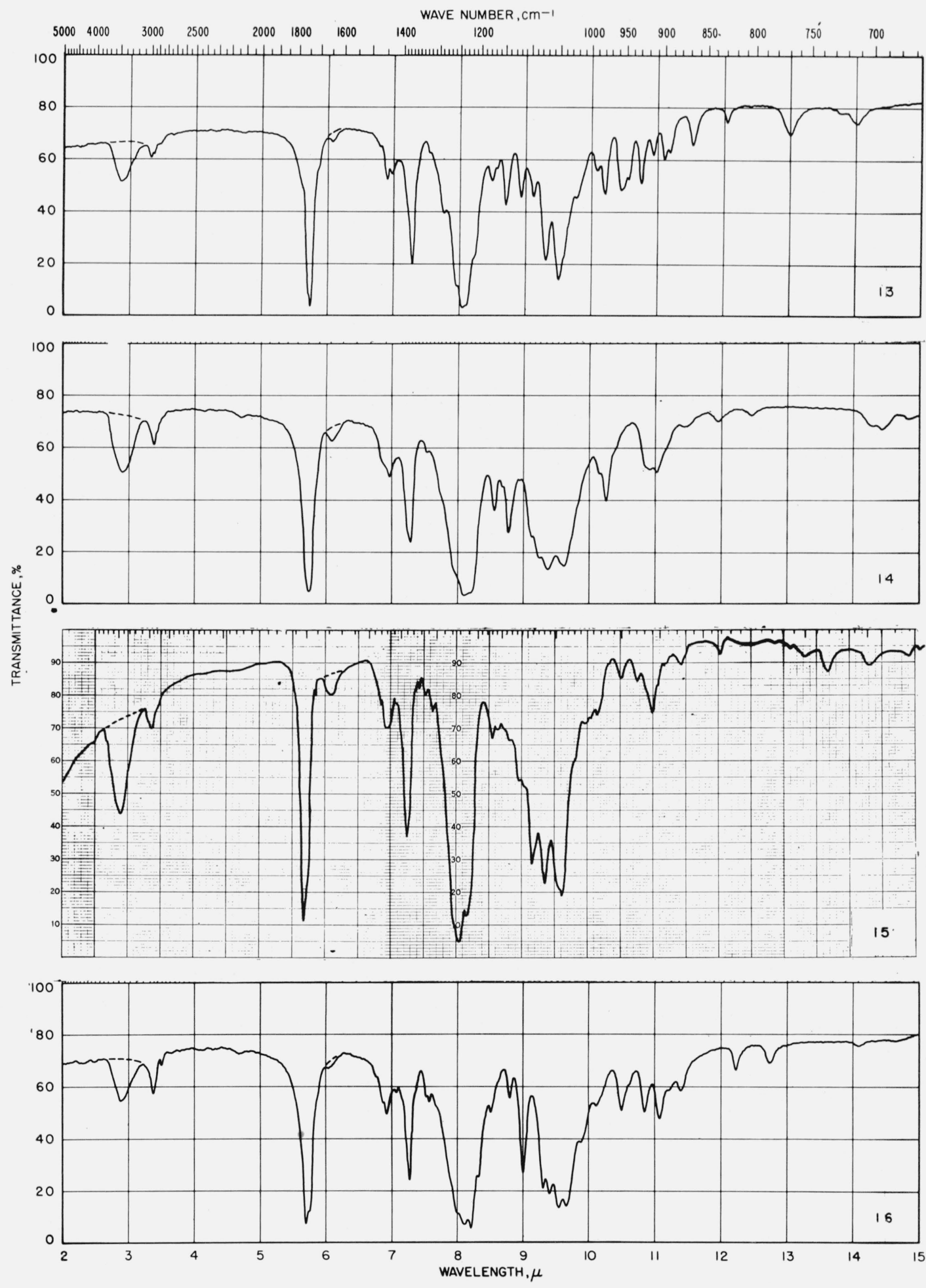

Figure 3. Spectrograms of materials in potassium chloride pellets.-Continued

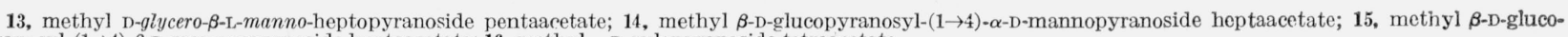
pyranosyl-(1-4)- $\beta$-D-mannopyranoside heptaacetate; 16. methyl $\alpha$-D-gulopyranoside tetraacetate. 

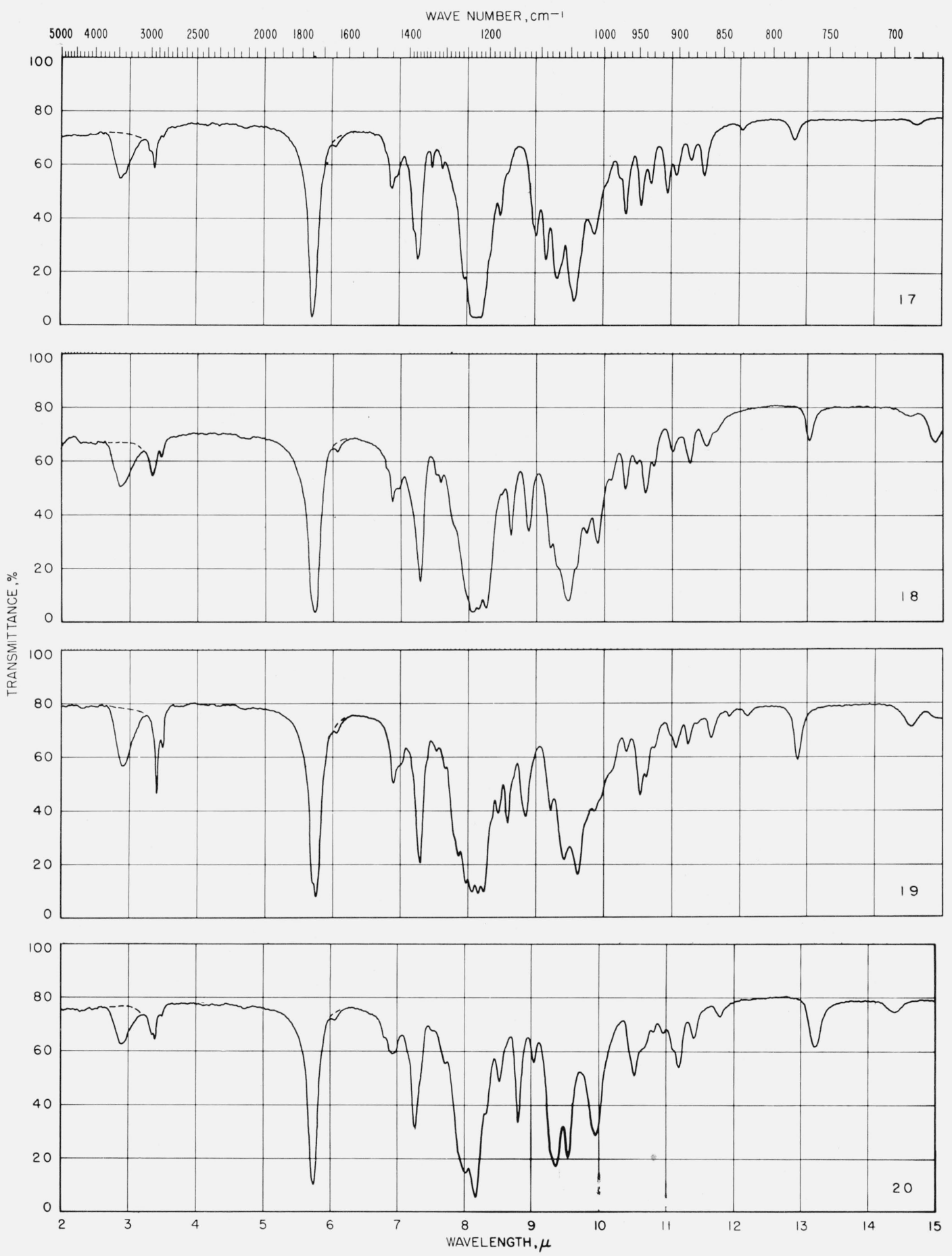

FIGURE 3. Spectrograms of materials in potassium chloride pellets.-Continued

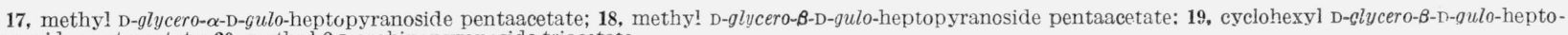
pyranoside pentaacetate; 20 , methyl $\beta$-I-arabinopyranoside triacetate. 

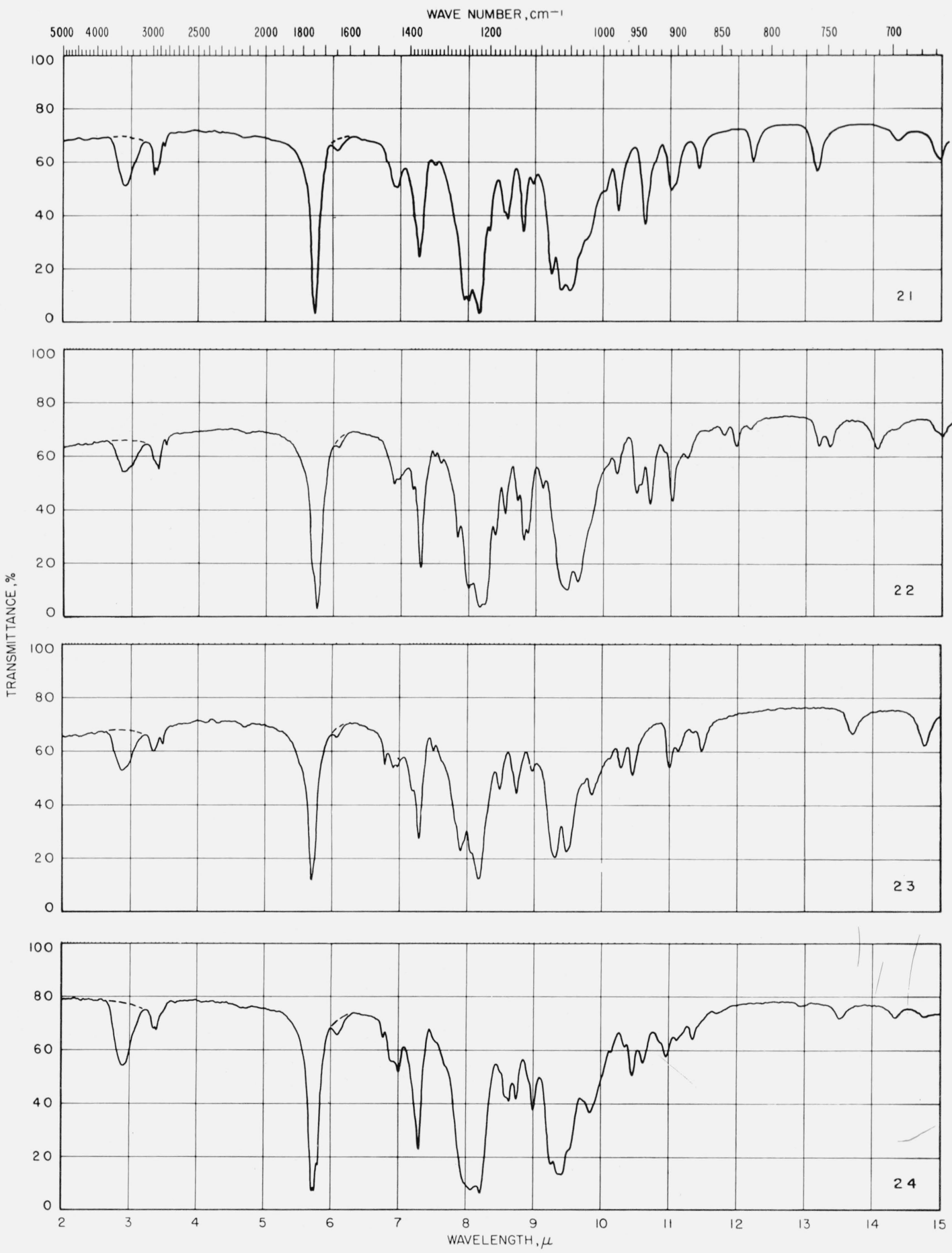

FiguRE 3. Spectrograms of materials in potassium chloride pellets.-Continued

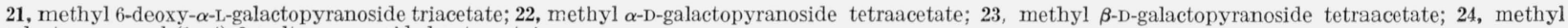
$\beta$-D-galactopyranosyl- $(1 \rightarrow 4)-\beta$-D-altropyranoside heptaacetate. 


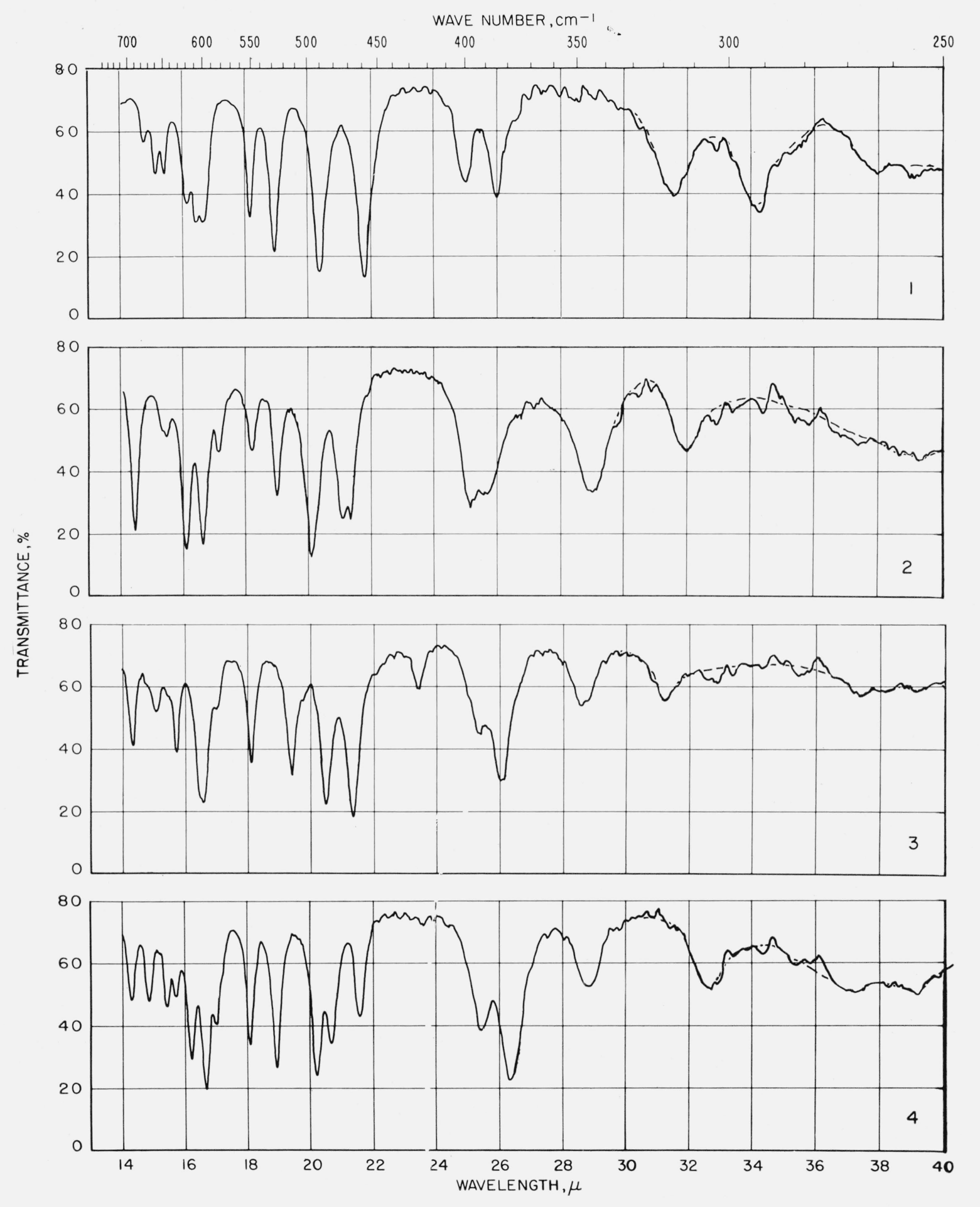

FIGURE 4. Spectrograms of materials in potassium iodide pellets.

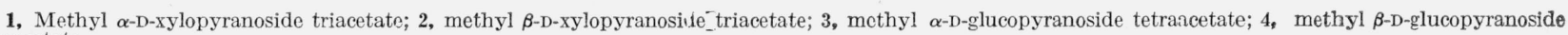
tetraacetate. 
WAVE NUMBER, $\mathrm{cm}^{-1}$
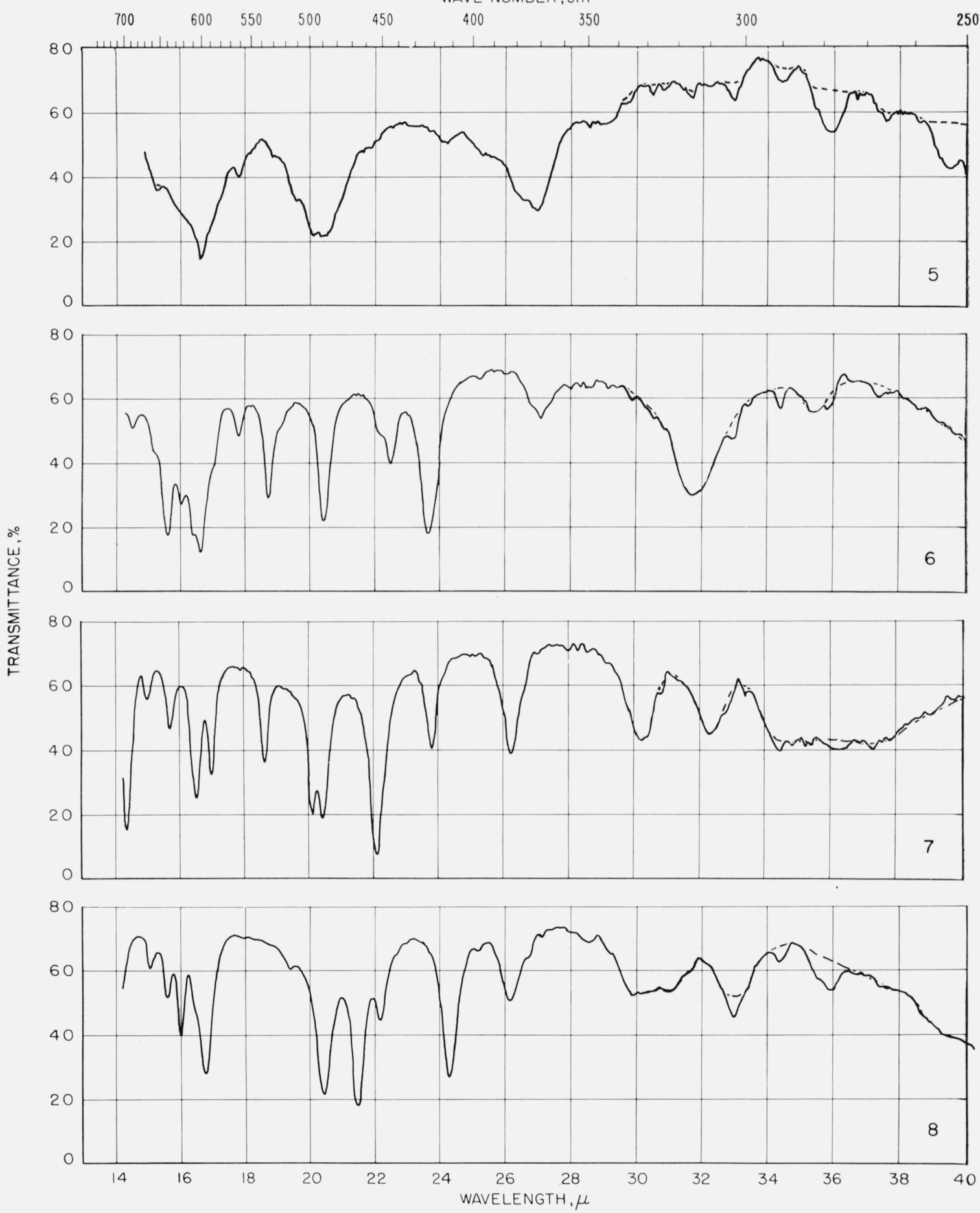

Figure 4. Spectrograms of materials in potassium iodide pellets.-Continued 

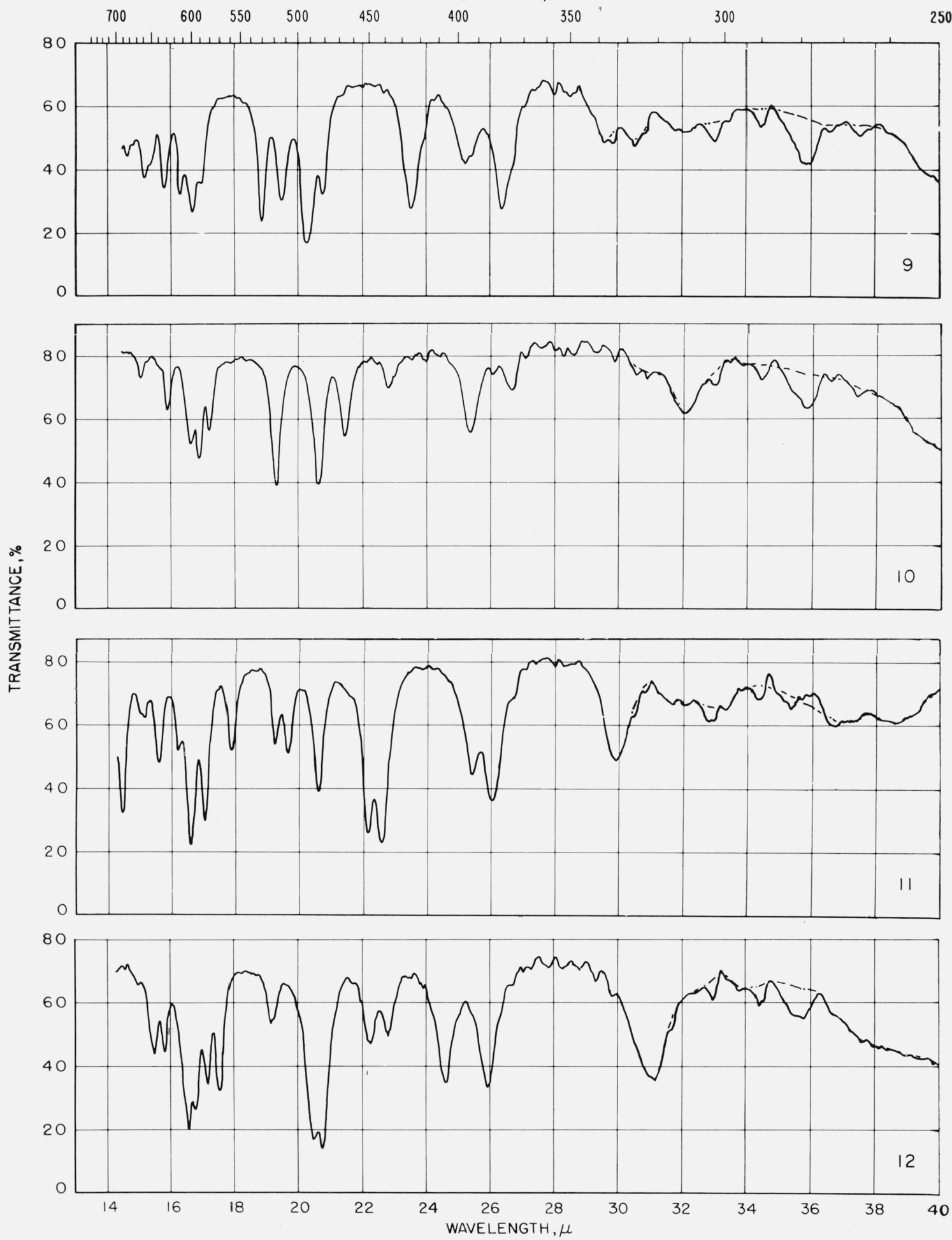

FIGURE 4. Spectrograms of materials in potassium iodide pellets.-Continued

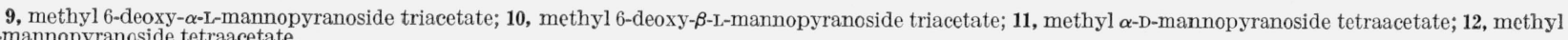
$\beta$-D-mannopyranoside tetraacetate. 
WAVE NUMBER, $\mathrm{cm}^{-}$
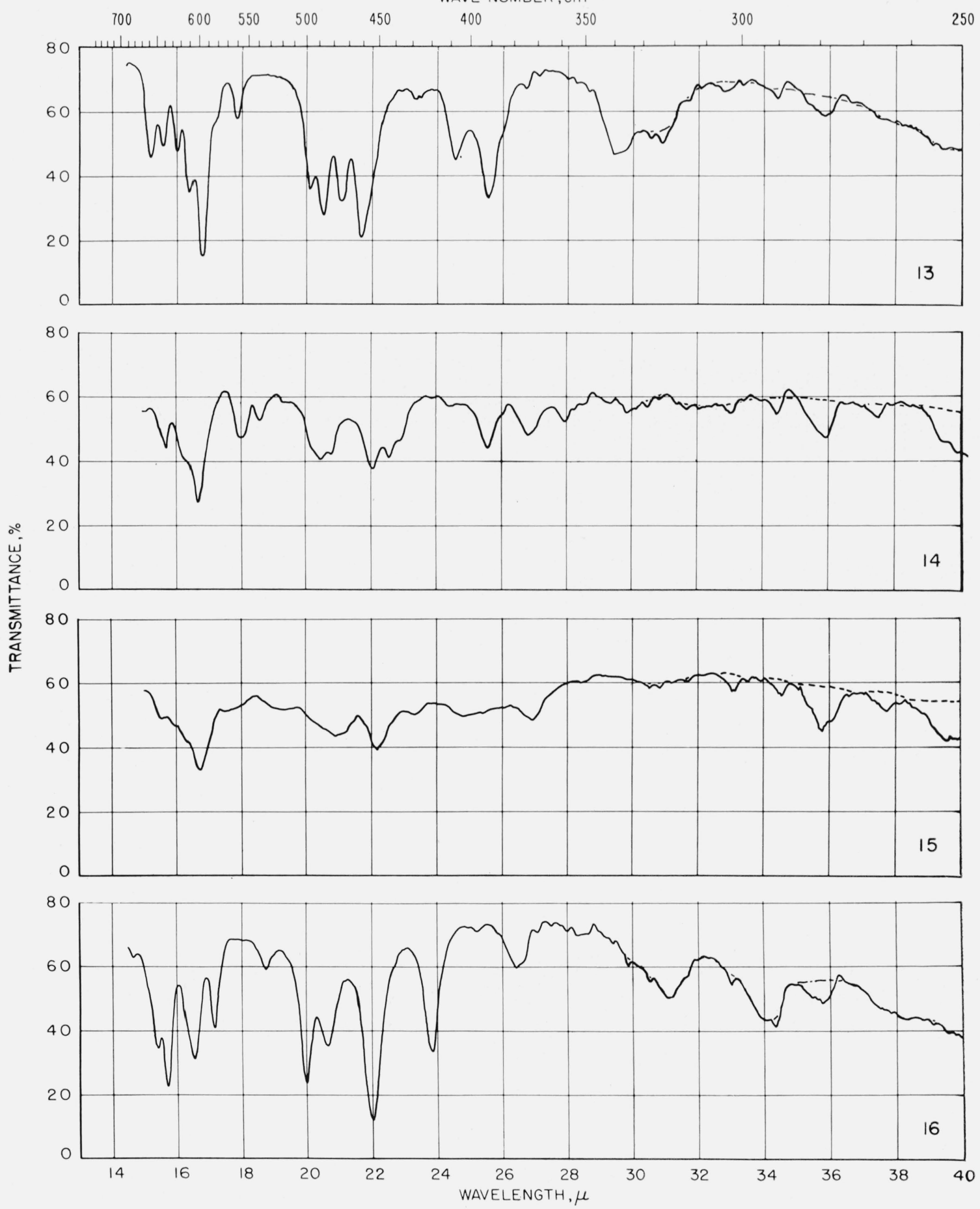

FIGURE 4. Spectrograms of materials in potassium iodide pellets.-Continued

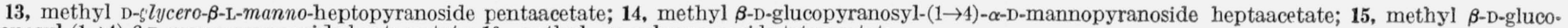
pyranosyl-( $1 \rightarrow 4)-\beta$-D-mannopyranoside heptaacetate; 16 , methyl $\alpha$-D-gulopyranoside tetraacetate. 

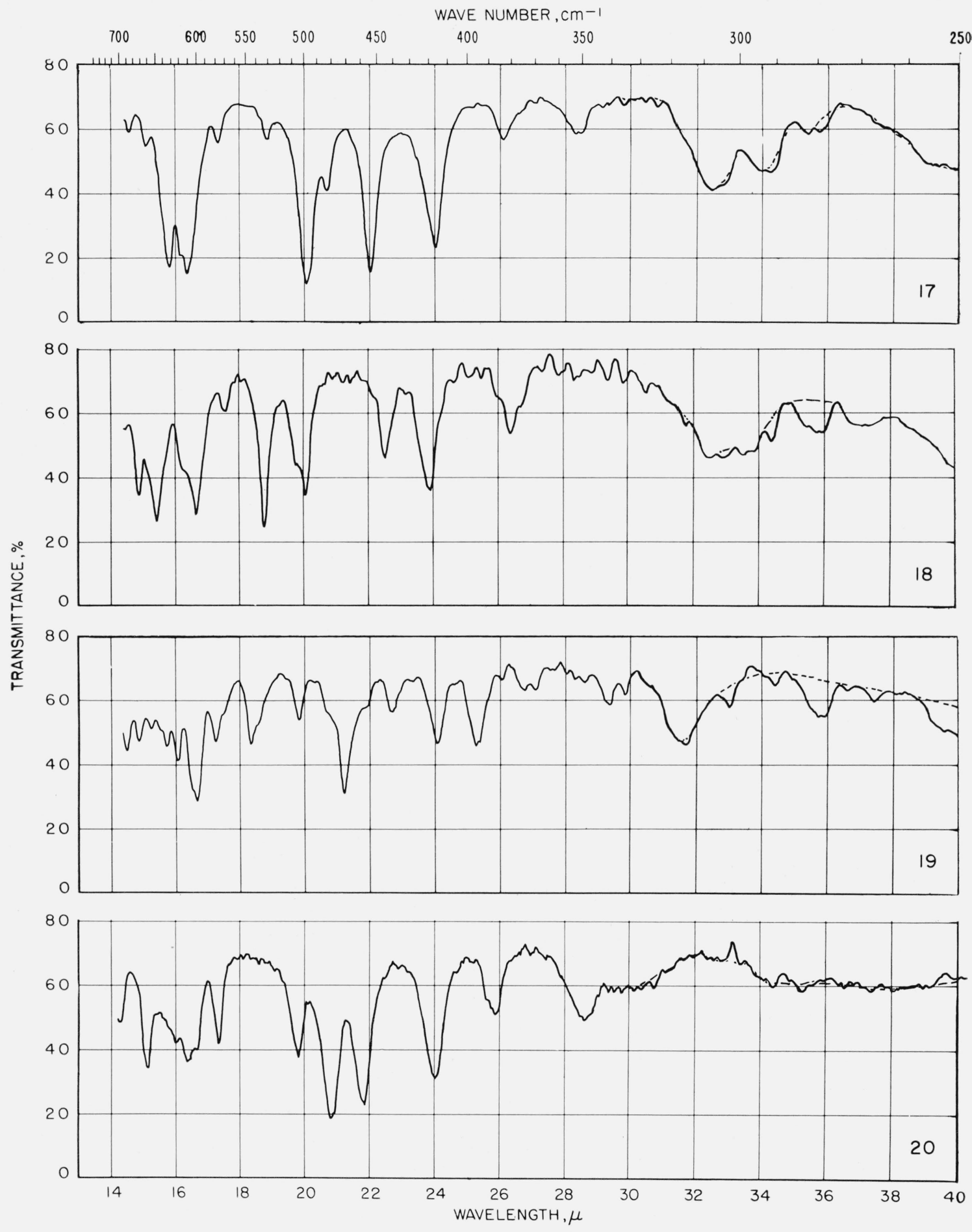

FIgURE 4. Spectrograms of materials in potassium iodide pellets.-Continued

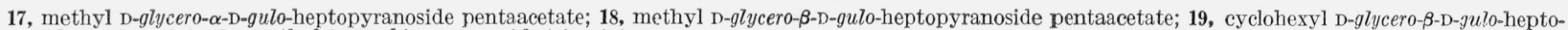
pyranoside pentaacetate; 20 , methyl $\beta$-L-arabinopyranoside triacetate. 
WAVE NUMBER, $\mathrm{cm}^{-1}$
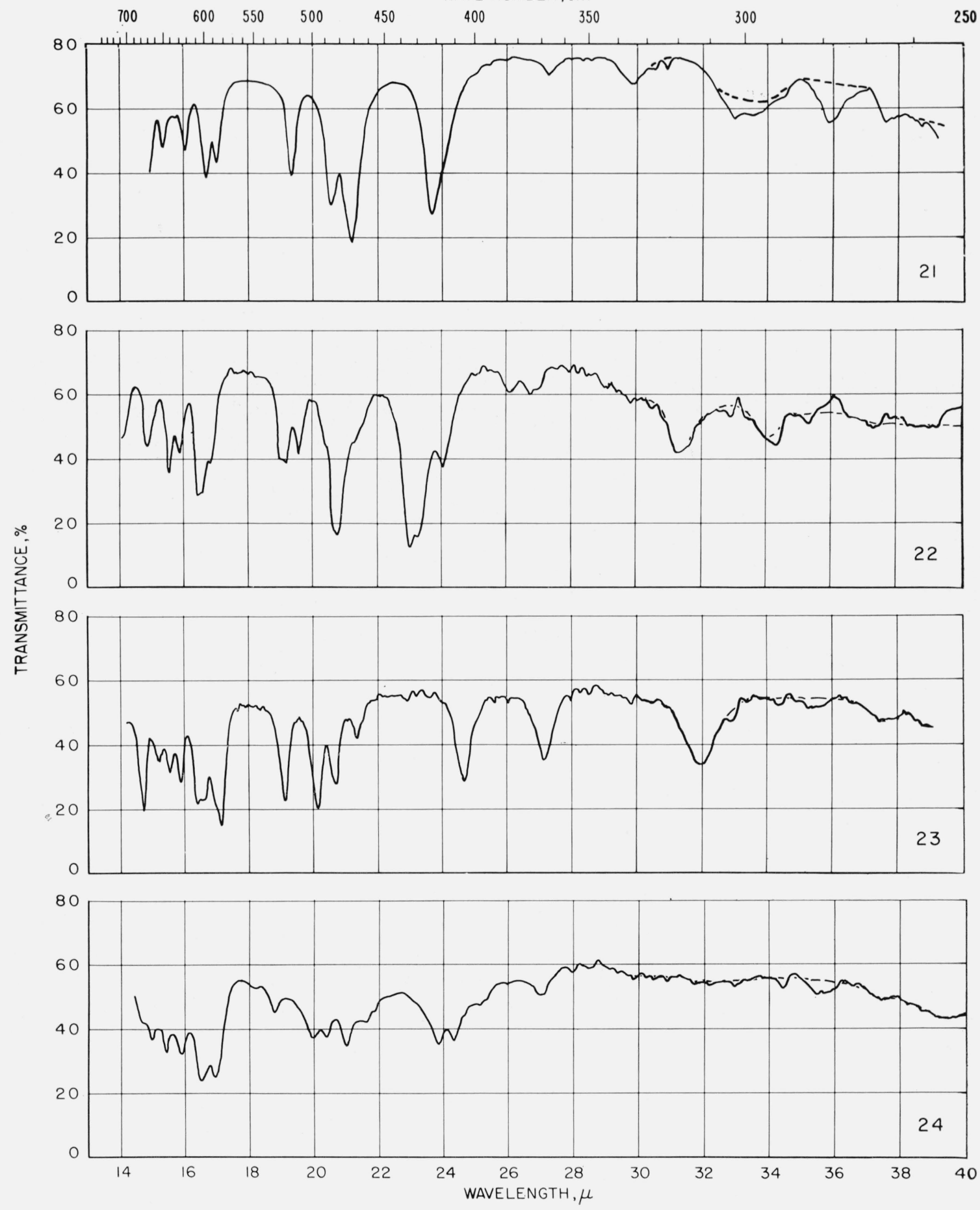

FIGURE 4. Spectrograms of materials in potassium iodide pellets.-Continued

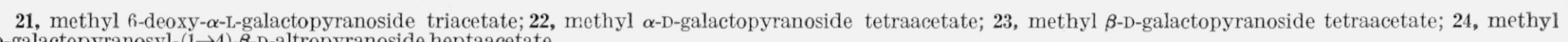

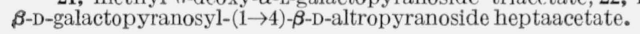




\subsection{Measurement of Infrared Absorption}

The spectrograms are shown in figures 3 and 4 . Those in figure 3 for compounds 5 and 15 were recorded with a Beckman Model IR4 (double-beam) spectrophotometer equipped with prisms of sodium chloride. The others were recorded with a PerkinElmer Model 21 (double-beam) spectrophotometer equipped with a prism of sodium chloride (for the range of 5,000 to $667 \mathrm{~cm}^{-1}$ ) and of cesium bromide (for the range of 667 to $250 \mathrm{~cm}^{-1}$ ), as previously described [11].

Some absorption attributable to water (in the compound, the alkali halide, or both) was observed at 3,448 and $1,639 \mathrm{~cm}^{-1}$ and, attributable to atmospheric water vapor, in the far-infrared curves. These regions are drawn on the spectrograms with dashed lines which merely indicate uncertainty and are not to be interpreted quantitatively.

\subsection{Spectra Measured Under Different Conditions}

The spectra of 13 of the acetylated aldopyranosides (compounds 1 to 4, 7, 9 to 12, 18, 20, 22, and 23) had previously been measured [3] in carbon tetrachloride and in either carbon disulfide, dioxane, or chloroform. As has previously been mentioned, the infrared absorption spectra of crystalline materials show more bands than the spectra of the same compounds in solution. As a result, a larger number of bands were available for correlations than in the previous study [3].
The authors express their gratitude to Harriet L. Frush and J. D. Moyer for preparing and purifying many of the compounds used in this study, and to J. E. Stewart, J. J. Comeford, and F. P. Czech for recording the infrared absorption spectra.

\section{References}

[1] L. P. Kuhn, Anal. Chem. 22, 276 (1950).

[2] H. S. Isbell, F. A. Smith, C. Creitz, J. D. Moyer, and H. L. Frush, Infrared spectra of sugar acetates and related compounds (informal communication).

[3] H. S. Isbell, F. A. Smith, E. C. Creitz, H. L. Frush, J. D. Moyer, and J. E. Stewart, J. Research NBS 59, 41 (1957) RP2772.

[4] R. L. Whistler and L. R. House, Anal. Chem. 25, 1463 (1953).

[5] S. A. Barker, E. J. Bourne, R. Stephens, and D. H. Whiffen, J. Chem. Soc. 1954, 3468 .

[6] R. S. Tipson and H. S. Isbell, J. Research NBS $64 \mathbf{A}$, 239 (1960).

[7] R. E. Reeves, J. Am. Chem. Soc. \%1, 215, 1737 (1949); \%2, 1499 (1950).

[8] H. S. Isbell, J. Research NBS 5\%, 171 (1956) RP2707.

[9] H. S. Isbell and R. S. Tipson, Science 130, 793 (1959).

[10] H. S. Isbell and R. S. Tipson, J. Research NBS $64 \mathbf{A}, 171$ (1960)

[11] R. S. Tipson, H. S. Isbell, and J. E. Stewart, J. Research NBS 62, 257 (1959) R P2960.

[12] H. B. Henbest, G. D. Meakins, B. Nicholls, and A. A. Wagland, J. Chem. Soc. 195\%, 1462.

[13] J. E. Page, J. Chem. Soc. 1955, 2017.

[14] H. S. Isbell and H. L. Frush, J. Research NBS 24, 125 (1940) RP1274.

Washington, D.C.

(Paper 64A5-65) 